\title{
The electrical double layer for a fully asymmetric electrolyte around a spherical colloid: an integral equation study
}

\author{
G. Iván Guerrero-García, ${ }^{1}$ Enrique González-Tovar, ${ }^{1,2}$ Marcelo Lozada-Cassou, ${ }^{2}$ and F. de J. Guevara-Rodríguez ${ }^{2}$ \\ ${ }^{1}$ Instituto de Física, Universidad Autónoma de San Luis Potosí, \\ Álvaro Obregón 64, 78000 San Luis Potosí, S.L.P., México \\ ${ }^{2}$ Programa de Ingeniería Molecular, Instituto Mexicano del Petróleo, \\ Eje Central Lázaro Cárdenas 152, 07730 México, D.F., México
}

(Dated: March 15, 2021)

\begin{abstract}
The hypernetted chain/mean spherical approximation (HNC/MSA) integral equation is obtained and solved numerically for a totally asymmetric primitive model electrolyte around a spherical macroparticle. The ensuing radial distribution functions show a very good agreement when compared to our Monte Carlo and molecular dynamics simulations for spherical geometry and with respect to previous anisotropic reference HNC calculations in the planar limit. We report an analysis of the potential vs charge relationship, radial distribution functions, mean electrostatic potential and cumulative reduced charge for representative cases of $1: 1$ and 2:2 salts with a size asymmetry ratio of 2. Our results are collated with those of the Modified Gouy-Chapman (MGC) and unequal radius Modified Gouy-Chapman (URMGC) theories and with those of HNC/MSA in the restricted primitive model (RPM) to assess the importance of size asymmetry effects. One of the most striking characteristics found is that, contrary to the general belief, away from the point of zero charge the properties of an asymmetric electrical double layer (EDL) are not those corresponding to a symmetric electrolyte with the size and charge of the counterion, i.e. counterions do not always dominate. This behavior suggests the existence of a new phenomenology in the EDL that genuinely belongs to a more realistic size-asymmetric model where steric correlations are taken into account consistently. Such novel features can not be described by traditional mean field theories like MGC, URMGC or even by enhanced formalisms, like HNC/MSA, if they are based on the RPM.
\end{abstract}

PACS numbers: 61.20.-p, 61.20.Gy, 61.20.Ja, 61.20.Qg.

\section{INTRODUCTION}

The electrical double layer (EDL) is the structure formed by electrolyte ions around a charged surface, usually that of a colloid or electrode. An understanding of the EDL properties is a crucial matter for science and technology because of the large variety of related applications, that range from colloidal stability, electrokinetics and the description of biological systems to daily manufactured products as inks, paint emulsions, foods or medicaments [1, 2]. As an illustration, the EDL determines completely the value of the zeta potential of a colloid in electrophoretic motion [3, 4]. The zeta potential, which is directly related to the measured mobility in electrophoresis experiments, is a central quantity in colloid science and many standard techniques of characterization, stabilization and separation of colloidal suspensions rely critically on its knowledge [5, 6, 6, [8]. Depending on the form of the dispersed macroparticle (or electrode) is that we are dealing with an EDL of particular geometry, e.g. planar, spherical or cylindrical. The planar case is, by far, the most studied instance, however, the spherical EDL (SEDL) deserves special attention for its obvious relation to dispersions of globular proteins, micelles, polymer beads, dendrimers or many other nearly spherical organic or inorganic macroions [1, 2, 5, [6, 7].

Since the emergence of this topic, the unquestionable relevance of the EDL has been paralleled by an intense search of an adequate theoretical description of such charge distribution. In order to build a successful theory of the EDL, a good model is essential. Among some more refined representations, nowadays, the usual election for the EDL is the minimal but able restricted primitive model (RPM). In this well-known idealization of a single EDL a "colloid" or "electrode" is mimicked by a rigid and uniformly charged object, which is immersed in an electrolyte solution constituted by a structureless solvent media and various ionic species, where the latter are treated as equally-sized hard spheres with punctual charges at their centers. To avoid image effects it is customary to suppose a uniform dielectric constant permeating all the space. During the past decades the RPM-EDL has been studied comprehensively via modern statistical mechanics approaches, namely integral equations $9,10,11,12$, 13, 14, 15, 16, 17, 18, 19, 20, 21, 22, 23., density functional theories 24, 25, 26, 27, 28, 29, 30, 31, 32, 33] and mean electrostatic potential schemes [34, 35, 36, 37]. Additionally, the usage of simulation techniques to evaluate the properties of these coulombic systems has supplied a large body of "experimental" data useful to test the existing theories 10, 38, 39, 40, 41, 42, 43, 44, 45, 46, 47]. At the end, and on spite of its apparent simplicity, all the collected evidence sustains the RPM as a concise and thriving model which, by embodying the most important interactions in EDL systems, i.e. electrostatic and hard-core potentials, is capable to provide not only an essentially correct account of the associated thermodynamic properties but also to predict characteristic phe- 
nomena of the EDL such as layering, charge inversion, charge reversal and overcharging. Briefly, we remember to the reader that the stratified (oscillating) ionic distributions next to a colloid are referred to as layering. On the other hand, charge reversal means the excessive compensation of the native colloidal charge, prompted by strongly attracted counterions, leading to an effective macroparticle's charge of reversed sign. This counterions layer, in turn, promotes a layer of coions adsorption which, consequently, produces charge inversion, i.e. a layer of inverted charge. Complementarily, overcharging is the unusual adsorption of coions to the surface of the colloid, which increases its original charge [48]. In fact, the continued progress of theories founded on the RPM has made of them a tool of quantitative capacity to interpret experimental results. As an example, very recently, the new Primitive Model Electrophoresis approach [49, 50] has incorporated consistently the ionic size effects into the electrokinetic equations and forged an enhanced treatment of electrophoresis, which fits correctly measurements in multivalent electrolytic ambients and explains reversed mobilities 49, 50, 51, 52].

Historically, the RPM-based theoretical attempts surged as to better the classical Poisson-Boltzmann (PB) portrait of the EDL. In the conventional PB treatment, instead of hard-sphere ions, a punctual electrolyte is assumed and the ionic distributions are obtained by solving the Poisson-Boltzmann differential equation, thus eliminating the very important interionic steric correlations present in the RPM. Although the assumption of point-ions could be acceptable in some cases (e.g., if low charged colloids in very diluted univalent salts are being considered), for high-coupled systems the inadequacy of such hypotesis has been clearly attested [12, 13, 16, 39, 53, 54]. As a consequence, the original bare PB theory is unable to predict any of the nonmonotonic characteristics mentioned in the previous paragraph. Since its early stages, the patent faults of the $\mathrm{PB}$ equation were already noticed [55, 56, 57, 58. and, accordingly, a first modification to the simple $\mathrm{PB}$ treatment was devised by introducing the concept of the Stern layer or the Helmholtz plane. In this supplemented model, the only inclusion of a unique distance of closest approach between the ions and the macroparticle resulted in the normalization of the otherwise unphysical values of the radial distribution functions (RDFs) close to the contact and, eventually, extended the applicability of the PB-Stern or modified Gouy-Chapman theory (PB-S or MGC) to 1:1 systems with moderate charge or concentration [10, 59]. Nevertheless, MGC remained exempt of nonmonotonical features and, thus, unsuccessful to cope with more demanding conditions (viz. multivalent and/or highly concentrated electrolytes or, else, high surface charges).

Up to now, most of the theoretical work on EDL has supposed the valence as the unique source of ionic asymmetry. Obviously, in nature this is not the situation and, out of the many possible sophistications to the model (e.g. a discrete solvent, more species, etc), the consideration of ions with different sizes is a first choice. Some works have been already published along these lines 60, 61, 62, 63, 64, 65, 66, 67] but, notably, the pioneering attempt to include distinct ionic sizes was formulated inside the framework of the PB equation by Valleau and Torrie [60], who used Stern layers of unequal extent for the counter- and coions. It must be noted that in this unequal radius MGC (URMGC) scheme the ions are, at the same time, voluminous and punctual objects, i.e., an electrolytic particle behaves as a hard sphere when interacting with the colloid (being dissimilar the coion and counterion distances of closest approach to the macroparticle), but among ions they "see" each other as points. In summary, such addition to MGC was rewarding since exposed interesting effects not observed in the size-symmetric case, e.g. the displacement of the potential of zero charge (PZC), the occurrence of oscillations in the radial distribution functions and the apparent dominance of counterions to determine the properties of asymmetric EDL systems. At first, all these phenomena seemed appealing since, for instance, the non-zero PZC allowed the possibility to interpret experimental data by obviating specific adsorption, and the occurrence of fluctuating URMGC ionic densities and electrostatic potentials meant that a "point-ion" theory should not be necessarily uniform. Furthermore, for a $n: n$ electrolyte with a common counterion size, the convergence between the MGC and URMGC outcomes was so clear that founded the now widely accepted fact that the counterion size is what really matters for the EDL. Quoting Valleau and Torrie: "...we expect the double layer properties of a dilute (asymmetric) electrolyte to become similar to those of a completely symmetric electrolyte having an effective size equal to that of the counterion. (This remark will be asymptotically exact for large fields in the PoissonBoltzmann theory)..." 60]. Notwithstanding, due to the absence of steric interionic correlations, all the new features in URMGC are constrained in space and can be rationalized purely in terms of energetic arguments, leaving out determinant entropic contributions [48, 68, 69], so that, at the end, URMGC will be incapable to produce a full description of the phenomenology happening in general size-asymmetric systems. Even more, we will make evident that an analysis based on the $\mathrm{PB}$ viewpoint of a totally asymetric EDL not only is partial but, regarding the counterion predominance, can lead in wrong directions.

For all the stated, to improve the theory of EDL the asymmetry in ionic size must be included in a more consistent way. That can be accomplished by means of the unrestricted primitive model (PM) of an electrolyte, for which the condition of equal size and charge in the RPM is relaxed. Not surprisingly, in the last years, the PMEDL for plane electrodes has been studied through the avenues of integral equations and the mean electrostatic potential [64, 65, 66]. Amid them, the Greberg and Kjellander's paper [66] represents a valuable contribution for 
its detailed appraisal of the effects of size asymmetry on the ionic structure and, chiefly, for its thorough discrimination of the different contributions to charge inversion and to the mean force. In any case, the body of existent literature on PM-EDL shares two limitations, namely they have dealt exclusively with the planar geometry, ignoring the undoubtfully notable spherical instance [70], and have focused on the structural properties or limited themselves to describe semiquantitatively the behavior of the potential versus charge relationship. Besides, and most importantly, due to the conditions explored, the majority of modern studies going beyond PB [10, 28, 32, 63, 64, 67, 71] have subscribed the wonted conclusion that the counterion-macroparticle interaction is what determines the EDL properties: "...Away from the pzc, the double layer is dominated by the counterions and, for a binary system at least, the properties of the double layer are essentially those of a symmetric salt with the charge and diameter of the counterion..." 63]. We will show that this is not always the case.

Consequently, in the present paper we report a comprehensive investigation of the PM-EDL for a spherical model colloid using integral equations and simulation techniques with a two-folded aim: i) to produce a description of the significant PM-SEDL system using a trustable statistical mechanics treatment and ii) to reivindicate the importance of size asymmetry in EDL studies in order to get a more faithful picture of a model colloidal suspension and as the origin of an enriched phenomenology, previously unseen in the symmetrical conditions. The rest of the paper is organized as follows. In Section II (and Appendices) we describe the model and give the details of the HNC/MSA theory for the PM-SEDL and of the employed simulation methods. The results, comparisons and their discussion are included in Section III, and we close in Section IV with our conclusions and some prospectives for future work.

\section{THEORY AND METHODS}

\section{A. The Basic Model}

Our representation of the SEDL is constituted by a rigid, charged spherical colloid of diameter $D$ and surface charge density $\sigma_{0}$, surrounded by a continuum solvent of dielectric constant $\epsilon$. The macroion is in contact with two ionic species which are treated as hard spheres of diameter $R_{i}(i=1,2)$ with embedded point charges of valence $z_{i}$ at their centers. It is assumed for definiteness that $R_{2} \geq R_{1}$. The interaction potential between the macroion and an ion of type $i$ is then given by

$$
U_{M i}(r)=\left\{\begin{array}{cc}
\infty, & r<\frac{D+R_{i}}{2}, \\
\frac{z_{i} e 4 \pi\left(\frac{D}{2}\right)^{2} \sigma_{0}}{\epsilon r}, & r \geq \frac{D+R_{i}}{2}
\end{array}\right.
$$

with $e$ the protonic charge. In turn, the interionic potential is

$$
U_{i j}(r)=\left\{\begin{array}{cl}
\infty, & r<\frac{R_{i}+R_{j}}{2} \\
\frac{z_{i} z_{j} e^{2}}{\epsilon r}, & r \geq \frac{R_{i}+R_{j}}{2} .
\end{array}\right.
$$

The Stern layer or, more properly, the Helmholtz plane, already mentioned in the introduction, is the geometrical place corresponding to the closest approach distance for the electrolyte ions to the colloid. If we consider an electrolyte formed by a pair of ionic species of unequal sizes, the closest approach distance to the surface for the smallest component, $\left(D+R_{1}\right) / 2$, determines the inner Helmholtz plane (IHP), whereas the corresponding distance for the largest species, $\left(D+R_{2}\right) / 2$, establishes the outer Helmholtz plane (OHP). In the limit of identical sizes the IHP and OHP coincide and the usual definition of the Helmoltz plane is recovered.

\section{B. The HNC/MSA equation for the PM-SEDL}

In general, the Ornstein-Zernike equation and the hypernetted chain closure (HNC) for a multicomponent mixture of $M$ species are

$$
h_{i j}\left(r_{12}\right)=c_{i j}\left(r_{12}\right)+\sum_{l=1}^{M} \rho_{l} \int h_{i l}\left(r_{13}\right) c_{l j}\left(r_{32}\right) d V
$$

and

$$
\begin{gathered}
c_{i j}\left(r_{12}\right)=-\beta U_{i j}\left(r_{12}\right)+h_{i j}\left(r_{12}\right)-\ln \left(h_{i j}\left(r_{12}\right)+1\right), \\
i, j=1,2 \ldots M,
\end{gathered}
$$

where $h_{i j}\left(r_{12}\right)$ is the total correlation function for particles 1 and 2 of species $i$ and $j$, distant by $r_{12}$, which is related to the radial distribution function by $g_{i j}\left(r_{12}\right)=$ $h_{i j}\left(r_{12}\right)+1, c_{i j}\left(r_{32}\right)$ is the direct correlation function for the pair 2 and $3, \rho_{i}$ is the bulk number concentration of each one of the species, $U_{i j}\left(r_{12}\right)$ is the direct interaction potential, $d V=d^{3} r_{13}$ is the differential volume, and $\beta=1 /\left(k_{B} T\right)$, where $k_{B}$ is the Boltzmann's constant and $T$ the absolute temperature.

Applying the Direct Method 72, 73, 74], let the species $M$ correspond to macroions at infinite dilution in a binary electrolyte. Then Eqs. (3) and (4) for species $M$ and $j$ become

$$
h_{M j}\left(r_{12}\right)=c_{M j}\left(r_{12}\right)+\sum_{l=1}^{2} \rho_{l} \int h_{M l}\left(r_{13}\right) c_{l j}\left(r_{32}\right) d V
$$

and

$c_{M j}\left(r_{12}\right)=-\beta U_{M j}\left(r_{12}\right)+h_{M j}\left(r_{12}\right)-\ln \left(h_{M j}\left(r_{12}\right)+1\right)$, 


$$
j=1,2 \text {. }
$$

If the HNC closure, Eq. (6), is used for the macroionion direct correlation function in the Ornstein-Zernike equation (5), we get

$$
g_{M j}(r)=\left\{\begin{array}{cc}
0, & r<\frac{D+R_{j}}{2}, \\
\exp \left\{-\beta U_{M j}(r)+\Omega_{M j}(r)\right\}, & r \geq \frac{D+R_{j}}{2},
\end{array}\right.
$$

with

$$
\Omega_{M j}(r)=\sum_{l=1}^{2} \rho_{l} \int\left(g_{M l}(t)-1\right) c_{l j}(s) d V
$$

where $r=r_{12}, t=r_{13}$ and $s=r_{23}=|\vec{t}-\vec{r}|$ are the distances between particles 1 and 2, 1 and 3, and 2 and 3 , respectively, and $d V=t^{2} \operatorname{sen} \theta d \theta d \phi d t$ is the volume element in spherical coordinates. The geometry of the system is illustrated in Fig. 1]

It must be noted that the sizes of the macroion and of the ionic species are arbitrary, and the only demanded condition is the electrolyte bulk electroneutrality:

$$
\rho_{1} z_{1}+\rho_{2} z_{2}=0 .
$$

In respect to the ion-ion direct correlation functions, $c_{i j}(s)$, in the bulk MSA theory they can be written as

$$
c_{i j}(s)=c_{i j}^{i n t}(s)+c_{i j}^{e x t}(s) .
$$

These functions are dependent on the size and charge of the ionic species and on other parameters of the system such as the temperature and the dielectric constant, and are stated explicitly in the Appendix A.

Introducing the MSA direct correlation functions in Eq. (8), we arrive to the following nonlinear system of integral equations (the index $M$ is dropped because there is only one macroion present):

$$
\begin{aligned}
g_{1}(r)= & \exp \left\{-\beta U_{1}(r)+\rho_{1} \int g_{1}(t) c_{11}(s) d V-\right. \\
& \rho_{1} \int c_{11}(s) d V+\rho_{2} \int g_{2}(t) c_{21}(s) d V- \\
& \left.\rho_{2} \int c_{21}(s) d V\right\}, \quad r \geq \frac{D+R_{1}}{2},
\end{aligned}
$$

and

$$
\begin{aligned}
g_{2}(r)= & \exp \left\{-\beta U_{2}(r)+\rho_{1} \int g_{1}(t) c_{12}(s) d V-\right. \\
& \rho_{1} \int c_{12}(s) d V+\rho_{2} \int g_{2}(t) c_{22}(s) d V- \\
& \left.\rho_{2} \int c_{22}(s) d V\right\}, \quad r \geq \frac{D+R_{2}}{2} .
\end{aligned}
$$

From the specific $c_{i j}(s)$ definition and considering the electroneutrality restriction, Eqs. (11) and (12) can be recast in a more compact form as

$$
\begin{aligned}
g_{1}(r)= & \exp \left\{I_{0}(r)+\rho_{1} I_{1}(r)-\rho_{1} I_{2}(r)\right. \\
& \left.+\rho_{2} I_{3}(r)-\rho_{2} I_{4}(r)\right\}
\end{aligned}
$$

and

$$
\begin{aligned}
g_{2}(r)= & \exp \left\{H_{0}(r)+\rho_{1} H_{1}(r)-\rho_{1} H_{2}(r)\right. \\
& \left.+\rho_{2} H_{3}(r)-\rho_{2} H_{4}(r)\right\},
\end{aligned}
$$

with

$$
\begin{gathered}
I_{0}(r)=\frac{-z_{1} e 4 \pi\left(\frac{D}{2}\right)^{2} \sigma_{0}}{k_{B} T \epsilon}\left(\frac{1}{r}\right), \\
I_{1}(r)=\int g_{1}(t) c_{11}(s) d V,
\end{gathered}
$$

$$
I_{2}(r)=\int c_{11}^{i n t}(s) d V
$$

$$
I_{3}(r)=\int g_{2}(t) c_{21}(s) d V
$$

$$
I_{4}(r)=\int c_{21}^{i n t}(s) d V
$$

$$
H_{0}(r)=\frac{-z_{2} e 4 \pi\left(\frac{D}{2}\right)^{2} \sigma_{0}}{k_{B} T \epsilon}\left(\frac{1}{r}\right)
$$

$$
H_{1}(r)=\int g_{1}(t) c_{12}(s) d V
$$

$$
H_{2}(r)=\int c_{12}{ }^{i n t}(s) d V
$$

$$
H_{3}(r)=\int g_{2}(t) c_{22}(s) d V
$$

and

$$
H_{4}(r)=\int c_{22}{ }^{i n t}(s) d V
$$

The detailed form of these terms is given in the Appendix B. 
As a particular case, when $R_{1}=R_{2}$ in Eqs. (11) and (2), the equations (13) and (14) are reduced to the HNC/MSA expressions for the RPM-SEDL, deduced by González-Tovar and Lozada-Cassou [16]. If the interionic size correlations are neglected in the diffuse EDL but the closest approach distances between the macroion and the ionic species are conserved, by setting $R_{1}=R_{2}=0$ in Eq. (2) and letting $R_{1}$ and $R_{2}$ arbitrary in Eq. (1), it is found that $I_{2}(r)=I_{4}(r)=H_{2}(r)=H_{4}(r)=0$ and the integral version of the URMGC theory is obtained. Additionally, if $R_{1}=R_{2}=0$ in Eq. (2) and $R_{1}=R_{2}$ in Eq. (1), the HNC/MSA equations are reduced to the corresponding MGC equations in integral form.

Derived from the ionic profiles, two quantities of special interest are the mean electrostatic potential (MEP) and the integrated surface charge density, which, considering the electroneutrality condition, can be written as:

$$
\psi(r)=\frac{4 \pi e}{\epsilon} \int_{r}^{\infty} \sum_{i} g_{i}(t) \rho_{i} z_{i}\left[t-\frac{t^{2}}{r}\right] d t
$$

and

$$
\sigma(r)=-\frac{e}{r^{2}} \int_{r}^{\infty}\left[\sum_{i} g_{i}(t) \rho_{i} z_{i}\right] t^{2} d t
$$

respectively. Such quantities yield important parameters of the system when evaluated at specific positions. For example, if $r=D / 2$ Eqs. (25) and (26) become the MEP at the macroion's surface, $\psi_{0}$, and the native surface charge density of the macroion, $\sigma_{0}$, respectively:

$$
\psi_{0}=\frac{4 \pi e}{\epsilon} \int_{\frac{D}{2}}^{\infty} \sum_{i} g_{i}(t) \rho_{i} z_{i}\left[t-\frac{t^{2}}{\frac{D}{2}}\right] d t
$$

and

$$
\sigma_{0}=-\frac{e}{\left(\frac{D^{2}}{2}\right)} \int_{\frac{D}{2}}^{\infty}\left[\sum_{i} g_{i}(t) \rho_{i} z_{i}\right] t^{2} d t
$$

Furthermore, for $r=\left(D+R_{1}\right) / 2$ Eq. (25) corresponds to the MEP at the IHP, $\psi_{I H P}$, whereas for $r=\left(D+R_{2}\right) / 2$ the MEP at the OHP, $\psi_{O H P}$, is obtained, i.e.

$$
\psi_{I H P}=\frac{4 \pi e}{\epsilon} \int_{\frac{D+R_{1}}{2}}^{\infty} \sum_{i} g_{i}(t) \rho_{i} z_{i}\left[t-\frac{t^{2}}{\frac{D+R_{1}}{2}}\right] d t
$$

and

$$
\psi_{O H P}=\frac{4 \pi e}{\epsilon} \int_{\frac{D+R_{2}}{2}}^{\infty} \sum_{i} g_{i}(t) \rho_{i} z_{i}\left[t-\frac{t^{2}}{\frac{D+R_{2}}{2}}\right] d t .
$$

A fundamental quantity in electrokinetics is the zeta potential, $\zeta$. This potential has been conventionally associated with the MEP at the closest approach distance between an electrolyte and a charged surface 13, 16, 75, 76, 77, 78]. Following this convention, in a size-asymmetric or PM electrolyte $\zeta=\psi_{I H P}$, and when the ions are equally-sized (in the RPM) $\zeta=\psi_{I H P}=$ $\psi_{O H P}$.

The integrated surface charge density is a very convenient magnitude for infinite surfaces such as unbounded planes or cylinders. Nevertheless, for finite surfaces, as spheres, ellipsoids or spherocylinders, is useful to consider instead the cumulative reduced charge (CRC):

$$
\begin{aligned}
& Q^{*}(r)=\frac{Q(r)}{\left|Q_{0}\right|}=\frac{1}{4 \pi\left(\frac{D}{2}\right)^{2}\left|\sigma_{0}\right|} \\
& \left(4 \pi\left(\frac{D}{2}\right)^{2} \sigma_{0}+4 \pi \int_{\frac{D}{2}}^{r}\left[\sum_{i} g_{i}(t) \rho_{i} z_{i} e\right] t^{2} d t\right),
\end{aligned}
$$

where $Q(r)=4 \pi r^{2} \sigma(r)$. Beyond the macroparticle's surface this quantity corresponds to the total charge (native plus adsorbed) inside a sphere of radius $r$, normalized with the bare charge over the colloid. If $D / 2 \leq r \leq$ $\left(D+R_{1}\right) / 2$ the $\mathrm{CRC}$ is equal to one, whereas for $r \rightarrow \infty$ this quantity goes to zero because of the electroneutrality condition. The CRC has also the property of indicating charge reversal when $Q^{*}(r) Q_{0}<0$. Moreover, charge reversal and overcharging are conveniently quantified by $Q^{*}(r)$ as a fraction or multiple of the native bare charge compensated locally up to certain distance.

\section{Computational methods}

For a given value of $\sigma_{0}$, the set of nonlinear integral Eqs. (13) and (14) was numerically solved via the successive substitution (or Picard) method. In the past, this technique has been successfully applied to work out integral equations associated to similar coulombic fluids problems 12, 13, 32]. We have produced accurate results by continuing the iterative procedure until the Euclidean norm between consecutive input and ouput of the RDFs was less than some prescribed small number, guaranteeing an error inferior to $1 \%$. As an extra test of consistency, at the end of the Picard cycle, the final ionic profiles were used to check to what extent they fullfilled the total electroneutrality condition in the form of Eq. (28). In all our calculations $\sigma_{0}$ was recovered up to a $99.9 \%$ at least.

For comparison intentions, the static properties of some of our PM-SEDL samples were also computed by performing Monte Carlo (MC) and molecular dynamics (MD) simulations. In particular, we focused on 2:2, $0.5 \mathrm{M}$ electrolytes with size asymmetry ratio of 2 (characterized by the parameters listed in Table $\amalg$ bathing a macroion. This macroion was considered as a hard sphere of diameter $D=10 \AA$ with a charge $Z_{M} e$ located at its center, with $Z_{M}=8$ and $e$ the protonic charge, which correspond to a surface charge density $\sigma_{0}=0.407 \mathrm{C} / \mathrm{m}^{2}$. The solvent 
enters the model as a uniform dielectric with an aqueous value of $\epsilon=78.5$ at a temperature $T=298 \mathrm{~K}$. In order to avoid image potential effects the dielectric constant of the macroion and the ionic species was considered equal to the solvent. The simulations were carried out within a cubic box with one macroion fixed in the center, surrounded by the electrolyte. The number of ions for each species was adjusted to satisfy the electroneutrality condition over all system: $N_{1} Z_{1} e+N_{2} Z_{2} e+Z_{M} e=0$. The usual periodic boundary conditions with the minimum image convention for the ions were imposed to the simulation box, whose length $(L)$ was considered sufficiently large to neglect macroion-macroion interactions and to obtain stable profiles (the specific parameters used are listed in Table [1). Following Degrève et al. 40, 41], the use of such a sufficiently large simulation box for SEDL systems owning a plasma parameter $79,80,81,82$ $\Gamma_{p}<10$ makes unnecessary a treatment à la Ewald of the coulombic interactions. Precisely, in our study $\Gamma_{p}=3.1$.

The MC simulations were performed in the canonical ensamble using the standard Metropolis method [83, 84]. The macroion-ion and ion-ion interaction potentials are given by Eqs. (11) and (2), respectively. In the thermalization process were carried out $4 \times 10^{4} \mathrm{MC}$ cycles to get the system into equilibrium and $2 \times 10^{5} \mathrm{MC}$ cycles were performed in order to take the canonical average after the equilibration. The aceptance ratio used was 0.4.

In the MD simulations the numerical integration of the motion equations was accomplished through a reversible MD technique [85]. This method ensures numerical stability and is equivalent to the velocity Verlet algorithm. In order to produce data in a feasible way and to avoid the problem of an indefinable force at contact, a very steep but continuous interparticle potential in lieu of a hard core one was employed, i.e.

$$
w_{i j}(r)=\left\{\begin{array}{cc}
A\left[\left(\frac{R_{i j}}{r}\right)^{2 l}-2\left(\frac{R_{i j}}{r}\right)^{l}+1\right], & r \leq R_{i j}, \\
0, & r>R_{i j}
\end{array}\right.
$$

where $A=k_{B} T, l=30$ and $R_{i j}$ is the closest approach distance between any pair of particles either macroionion or ion-ion. In consequence, the total potential among charges is the sum of $w_{i j}(r)$ and the Coulomb contribution as defined in Eqs. (11) and (2). The time step used was $\Delta t=10^{-3} \tau$, where $\tau=\sqrt{m_{0} R_{1}^{2} /\left(k_{B} T\right)}$ is the unit of time and $m_{0}$ is a characteristic unit of mass. The inertial masses of the components of the system are $m_{1}=m_{0}$, $m_{2}=8.0 m_{0}$ and $m_{\text {macroion }}=13.03 m_{0}$. Finally, the formal runs involved more than $8 \times 10^{6}$ MD steps after equilibration.

\section{RESULTS AND DISCUSSION}

In order to assess the reliability of the HNC/MSA equation for the PM-SEDL a comparison of our re- sults with simulation data and, possibly, against alternative theoretical approaches is called for. In the first case, collating with computer experiments is very useful to discriminate the adequacy of an approximate theory for a given model system. Additionally, confronting HNC/MSA with another available and, supposedly, more robust formalisms allows us to test its degree of accuracy and economy.

Previously, it has been shown that HNC/MSA agrees very well with computer data for the RPM of planar, spherical and cylindrical EDLs with symmetrical or asymmerical valences 12, 14, 40, 41, 44, 45, 47, 86]. On these grounds, now, we have opted for a certainly strict comparison, namely for a divalent electrolyte with appreciable size asymmetry. The two available options for dissymmetry were considered: counterions smaller than coions and viceversa.

At this point, it is important to mention that even if the equations developed in the last section are sufficiently general to include asymmetry in size and charge, here we will restrict our attention to $n: n$ electrolytes (i.e. $\left|z_{+}\right|=$ $\left.\left|z_{-}\right|\right)$to centre on the effect of different sizes. Following this convention, thereinafter, when we refer to $P M$ we will only imply distinct ionic diameters.

In Fig. 2 (main panel) we present the radial distribution functions for $\mathrm{HNC} / \mathrm{MSA}$ and MC simulations for a macroion of diameter $D=10 \AA$ and surface charge density $\sigma_{0}=0.407 \mathrm{C} / \mathrm{m}^{2}$ in a $2: 2,0.5 \mathrm{M}$ electrolyte of diameters $R_{-}=4.25 \AA$ and $R_{+}=8.5 \AA$. The inset of Fig. 2] shows RDFs for the same system but collating HNC/MSA with MD data. The Fig. 3 contains a similar comparison between $\mathrm{MC}$ and MD simulations and the HNC/MSA theory for the same system considered in the Fig. 2] but now for a salt with interchanged ionic sizes, $R_{-}=8.5 \AA$ and $R_{+}=4.25 \AA$. From these evidences, it is rewarding the excellent concordance between theory and simulation for the two possible asymmetries.

Complementarily, we also made some comparisons between the spherical HNC/MSA in the limit $D \rightarrow \infty$ and published data of the anisotropic reference HNC theory (ARHNC) for a charged planar wall 66]. We resorted to this limit because, to the best of our knowledge, there are no alternate structural results for a charged macrosphere in a size-asymmetrical electrolyte 63. Furthermore, at least for the planar geometry, ARHNC is an accurate description that considers the inhomogeneous correlations in the electrolyte due to the wall and that fits very closely the simulations of the RPM-EDL. Thus, in Fig. 团 (main panel) are displayed the HNC/MSA ionic profiles for a huge macroion of diameter $D=1000 \times R_{-}$and charge density $\sigma_{0}=0.267 \mathrm{C} / \mathrm{m}^{2}$, surrounded by a $1: 1,1 \mathrm{M}$ electrolyte with $R_{-}=4.25 \AA$ and $R_{+}=8.5 \AA$, and the ARHNC RDFs corresponding to a charged wall bearing the same $\sigma_{0}$ and under identical electrolytic parameters. To cover a more exigent situation, in the inset of Fig. 4 we plot the HNC/MSA distributions of a 2:2, $1 \mathrm{M}$ electrolyte of ionic dimensions $R_{-}=4.25 \AA$ and $R_{+}=6.375$ $\AA$ around a large colloid, characterized by $D=1000 \times R_{-}$ 
and $\sigma_{0}=0.267 \mathrm{C} / \mathrm{m}^{2}$, along with the ARHNC outcomes for the same electrolyte in contact with a wall of equal $\sigma_{0}$. Clearly, for both the uni- and divalent systems, the coincidence between HNC/MSA and ARHNC is notable, considering that $\mathrm{HNC} / \mathrm{MSA}$ is a simpler and less computationally demanding theory. Summarizing, the comparisons with Monte Carlo, molecular dynamics and ARHNC prove the trustability of HNC/MSA for the PM-SEDL and gives us the confidence to explore its predictions in a wider range of conditions.

A global and concise manner to analize the properties of the EDL for a large set of different states is in terms of the relationship between the MEP at some some point and the surface charge. In this respect, the first charge derivative of the MEP at the surface, $\frac{d \psi_{0}}{d \sigma_{0}}$, is relevant because of its connection with the differential capacity 31, 87, 88, 89, 90, 91, 92], whereas the MEP at the IHP, $\psi_{I H P}$, is conventionally associated with the zeta potential, $\zeta$, a key quantity in electrokinetic phenomena. Besides, and as it will be shown, it is precisely in the behavior of these MEPs that the importance and extension of the size asymmetry effects under study are evidenced. Because of this, we have calculated the functions $\psi_{0}\left(\sigma_{0}\right)$ and $\psi_{I H P}\left(\sigma_{0}\right)$ for a size-asymmetric SEDL using the following four theoretical schemes: URMGC, MGC, $\mathrm{HNC} / \mathrm{MSA}$ in the PM $\left(\mathrm{HNC} / \mathrm{MSA}_{P M}\right)$, and $\mathrm{HNC} / \mathrm{MSA}$ in the RPM $\left(\mathrm{HNC} / \mathrm{MSA}_{R P M}\right)$. In a series of preliminary runnings we have noticed that the manifestations of size asymmetry are more emphatic when the counterions are smaller than coions, thus, below we shall concentrate on the case where the diameter of the coions is twice that of the counterions. For comparison purposes, the parameters of the systems are adjusted such as for URMGC and HNC/MSA $P M$ the unequal colloidcounterion and colloid-coion closest approach distances are shared, and for MGC and $\mathrm{HNC} / \mathrm{MSA}_{R P M}$ the unique closest approach distance corresponds to that employed in HNC/MSA ${ }_{P M}$ for colloid-counterion. Therefore, in the rest of our calculations when the ionic diameters of a PM electrolyte, $R_{-}$and $R_{+}$, are stated it is meant that the different theories are solved for the following elections of the colloid-ion and ion-ion distances of closest approach, $d_{i}$ and $d_{i j}$, respectively (with $\sigma_{0} \geq 0$ ):

$d_{i}= \begin{cases}d_{-}=\frac{D+R_{-}}{2} \text { and } d_{+}=\frac{D+R_{+}}{2}, & \text { for HNC/MSA } \text { and URMGC }_{P M} \\ d_{-}=d_{+}=\frac{D+R_{-}}{2}, & \text { for HNC/MSA } R P M \\ & \text { and MGC, }\end{cases}$

$d_{i j}= \begin{cases}d_{-+}=d_{+-}=\frac{R_{-}+R_{+}}{2}, & \text { for HNC/MSA } \operatorname{MAM}_{P M}, \\ d_{-+}=d_{+-}=R_{-}, & \text {for HNC/MSA } R P M \\ d_{-+}=d_{+-}=0, & \text { for URMGC and MGC }\end{cases}$

For the theories mentioned in the foregoing the dependence of $\psi_{0}$ and $\psi_{I H P}$ on the surface charge $\sigma_{0}$ is given in Figs. 5(a) and 5 (b), respectively, for a macroion with $D=160 \AA$ and $\sigma_{0} \geq 0$ and a $1: 1,1$ M electrolyte with $R_{-}=4.25 \AA$ and $R_{+}=8.5 \AA$. In those figures, for any non-zero value of $\sigma_{0}$, visible quantitative discrepancies are seen when the URMGC and $\mathrm{HNC} / \mathrm{MSA}_{P M}$ potential curves are contrasted, or when the same is done with the $\mathrm{MGC}$ and $\mathrm{HNC} / \mathrm{MSA}_{R P M}$ ones. Remarkably, the maximum coincidence between the pair of URMGC and HNC/MSA $P M$ curves of $\psi_{0}\left(\sigma_{0}\right)$ (and of $\psi_{I H P}\left(\sigma_{0}\right)$ ) occurs precisely at the point of zero charge. In a similar way, the MGC and $\mathrm{HNC} / \mathrm{MSA}_{R P M}$ values of $\psi_{0}$ (and of $\psi_{I H P}$ ) converge at $\sigma_{0}=0$, as it is known from the studies of the charge-symmetric RPM-EDL 10, 12, 13, 16 (for the charge-asymmetric case see [31, 67, 71, 86, 93]). On the other hand, for a given value of $\sigma_{0}$, the differences between the results of a Poisson-Boltzmannian theory and the corresponding non-punctual HNC/MSA equation for $\psi_{0}$ and $\psi_{I H P}$ grow when size asymmetry is taken into account, e.g. at $\sigma_{0}=0.1 \mathrm{C} / \mathrm{m}^{2},\left(\psi_{0}\right)^{M G C}-\left(\psi_{0}\right)^{H N C / M S A_{R P M}}=$ $10.04 \mathrm{mV}$ whereas $\left(\psi_{0}\right)^{U R M G C}-\left(\psi_{0}\right)^{H N C / M S A_{P M}}=$ $24.11 \mathrm{mV}$ (see Fig. [5(a)), and $\left(\psi_{I H P}\right)^{M G C}$ $\left(\psi_{I H P}\right)^{H N C / M S A_{R P M}}=10.04 \mathrm{mV}$ whereas $\left(\psi_{I H P}\right)^{U R M G C}-\left(\psi_{I H P}\right)^{H N C / M S A_{P M}}=24.12 \mathrm{mV}$ (see Fig. 5(b)). Also, from Fig. [5 it is confirmed that the potential curves of URMGC approach asymptotically to those of MGC when $\sigma_{0}$ increases, as first pointed out by Valleau and Torrie [60]. Contrastingly, the $\psi_{0}\left(\sigma_{0}\right)$ and $\psi_{I H P}\left(\sigma_{0}\right)$ curves obtained from $\mathrm{HNC} / \mathrm{MSA}_{P M}$ and $\mathrm{HNC} / \mathrm{MSA}_{R P M}$ exhibit a clear separation for all surface charges, which means that counterions do not dominate in the $S E D L$ or, else, that the size of the coions matters even for large $\sigma_{0}$. From these observations it is infered that: i) an essentially punctual theory (MGC or URMGC) is not valid to describe the SEDL under high coupling conditions in which the size of the ions is relevant for all their interactions (ion-ion or colloid-ion), except maybe in the neighborhood of $\sigma_{0}=0$, and, two important points, ii) that including size-asymmetry in the model of an EDL is determinant since it exacerbates the steric interionic effects previously found in the RPM-EDL and iii) that the properties of the EDL are not totally determined by the counterions.

Expectedly, it is found that all the referred phenomena detected in monovalent electrolytes are more pronounced for divalent ions, as it is evidenced in Figs. 6(a) and 6(b) where the associated $\mathrm{HNC} / \mathrm{MSA}_{P M}, \mathrm{HNC} / \mathrm{MSA}_{R P M}$, URMGC and MGC results for $\psi_{0}\left(\sigma_{0}\right)$ and $\psi_{I H P}\left(\sigma_{0}\right)$ are reported for a $2: 2,0.5 \mathrm{M}$ electrolyte with $R_{-}=4.25 \AA$ and $R_{+}=8.5 \AA$, around a macrosphere of diameter $D=$ $160 \AA$ and positive charge. For instance, now the discrepancies between the MEPs of PB and HNC/MSA have increased in such a way that even the potentials of zero charge, $\psi_{0}\left(\sigma_{0}=0\right)$ and $\psi_{I H P}\left(\sigma_{0}=0\right)$, for URMGC and $\mathrm{HNC} / \mathrm{MSA}_{P M}$ do not coincide. Once more, URMGC has MGC as a limit for $\sigma_{0} \rightarrow \infty$ whereas HNC/MSA $P M$ and $\mathrm{HNC} / \mathrm{MSA}_{R P M}$ do not merge, notwithstanding, this time the following very interesting feature shows up: the $\psi_{I H P}\left(\sigma_{0}\right)$ relationship for $\mathrm{HNC} / \mathrm{MSA}_{R P M}$ exhibits the 
usual behavior documented in prior studies of positively charged colloids in 2:2 solutions [16, 92], i.e. it begins positive, reaches a maximum and becomes negative only at very high surface charges $\left(\sigma_{0} \simeq 0.344 \mathrm{C} / \mathrm{m}^{2}\right)$, whilst, in contrast, $\psi_{I H P}\left(\sigma_{0}\right)$ for $\mathrm{HNC} / \mathrm{MSA}_{P M}$ behaves differently, that is, starts negative at low $\sigma_{0}$, then regains "normal" positive values, experiences a maximum and, finally, becomes negative again at $\sigma_{0} \simeq 0.22 \mathrm{C} / \mathrm{m}^{2}$, much before $\mathrm{HNC} / \mathrm{MSA}_{R P M}$ does. This new fact could be consequential in electrokinetics since the signs of the MEP at the IHP and of the electrophoretic mobility of a colloid, $\mu$, frequently coincide (due to the identification $\zeta=\psi_{I H P}$ ), from which it should be possible to observe experimentally a macroion in a multivalent medium having a reentrant mobility, i.e. with inversion of $\mu$ at low $\sigma_{0}$, a normal sign for intermediate charges, and a posterior return to a inverted $\mu$ for high $\sigma_{0}$.

A more detailed examination of the size asymmetry consequences, already discussed at the level of the potential-charge relationship, can be made in terms of the SEDL structure. Thence, in the next figures, Figs. 7 and 8. we proceed to analyze the behavior of the ionic profiles of univalent solutions described by the MGC, UR$\mathrm{MGC}, \mathrm{HNC} / \mathrm{MSA}_{R P M}$ and $\mathrm{HNC} / \mathrm{MSA}_{P M}$ formalisms. To establish a connection with our previous MEP curves, the RDFs were obtained for a 1:1, $1 \mathrm{M}$ electrolyte, with $R_{-}=4.25 \AA$ and $R_{+}=8.5 \AA$, dissolving a macroion of size $D=160 \AA$ and surface charges $\sigma_{0}=0.08356 \mathrm{C} / \mathrm{m}^{2}$ (in Fig. 7) and $\sigma_{0}=0.3004 \mathrm{C} / \mathrm{m}^{2}$ (in Fig. 8). For each colloidal charge, the $\mathrm{HNC} / \mathrm{MSA}_{R P M}$ and $\mathrm{HNC} / \mathrm{MSA}_{P M}$ data are compared in the main panels of the figures, meanwhile, in the insets the MGC and URMGC plots are collated, to evaluate separately the effect of varying $\sigma_{0}$ on the distributions $g_{i}(r)$ when size asymmetry is incorporated into $\mathrm{HNC} / \mathrm{MSA}$ or $\mathrm{PB}$ theories. From the insets it is manifest the almost complete convergence between the monotonic URMGC and MGC RDFs for growing $\sigma_{0}$, as it could be awaited from the preceding results for $\psi_{0}\left(\sigma_{0}\right)$ and $\psi_{I H P}\left(\sigma_{0}\right)$ 60]. In any case, some distinctions between the URMGC and MGC profiles must naturally persist for all $\sigma_{0}$ (with the only exception of the unphysical value $\sigma_{0}=\infty$ ), due to the severe condition $g_{2}(r)=0$ for $r<\left(D+R_{2}\right) / 2$. Such restriction demands a finite discontinuity in $g_{2}\left(\frac{D+R_{2}}{2}\right)$ for URMGC and thus, for $0 \leq \sigma_{0}<\infty$, impedes a perfect coincidence between the RDFs of URMGC and MGC in the vicinity of the colloid surface. In other words, the total equivalence of URMGC and MGC is possible just in the limit of infinite surface charge, since only then the infinite and predominant electrostatic repulsion from the macroparticle will force the coions in the MGC description to be completely absent from the region $\left(D+R_{1}\right) / 2 \leq r<\left(D+R_{2}\right) / 2$, even if their punctual nature would allow them to be there. To be more explicit, if we define the abbreviations $C_{-}=\left(D+R_{-}\right) / 2$ and $C_{+}=\left(D+R_{+}\right) / 2$, an inspection of the $1: 1$ counterion contact values, $g_{-}\left(C_{-}\right)$, in Table confirms the existence of small but non-zero differences between the URMGC and MGC RDFs close to the macroparticle. Moreover, an important piece of information arises from Table II and the insets of Figs. [7 and 8 for systems with $\sigma_{0} \geq 0$ and $R_{-}<R_{+}$when $\sigma_{0}$ is enlarged, namely that the URMGC counterion contact RDF tends uniformly to that of MGC from below $\left(g_{-}^{U R M G C}\left(C_{-}\right) \uparrow g_{-}^{M G C}\left(C_{-}\right)\right)$, and that the URMGC coion RDF at $C_{+}$goes uniformly to that of MGC from above $\left(g_{+}^{U R M G C}\left(C_{+}\right) \downarrow g_{+}^{M G C}\left(C_{+}\right)\right)$. In a more global way, it is noticed therein that, $\forall r$, $g_{-}^{U R M G C}(r) \uparrow g_{-}^{M G C}(r)$ and $g_{+}^{U R M G C}(r) \downarrow g_{+}^{M G C}(r)$ as the colloidal charge grows, or, equivalently, that the $U R$ $M G C$ and $M G C$ ionic profiles converge when $\sigma_{0} \rightarrow \infty$ but they never cross mutually.

On the other hand, the comportment of the RDFs for $\mathrm{HNC} / \mathrm{MSA}_{P M}$ and $\mathrm{HNC} / \mathrm{MSA}_{R P M}$ in the main panels of Figs. [7 and 8 contrasts with the $\mathrm{PB}$ picture at the insets. For our 1:1 electrolyte with counterions smaller than coions, the ionic distributions of $\mathrm{HNC} / \mathrm{MSA}$ in the PM and RPM already exhibit a nonmonotonic behavior and charge inversion (in the case of RPM these features are unapparent due to the scale). Besides, from those graphs we arrive to one of the main conclusions of this work, that size asymmetry enhances significantly the nonmonotonical characteristics (e.g. oscillations and charge inversion) of the $S E D L$. In relation with the conduct of the SEDL structure for high $\sigma_{0}$, the $H N C / M S A_{P M}$ ionic densities do not have the $H N C / M S A_{R P M}$ ones as a limit and, in fact, from Table Iit is seen that the contact values of the counterion $\mathrm{RDF}$ in $\mathrm{HNC} / \mathrm{MSA}_{P M}$ exceed appreciably those of $\mathrm{HNC} / \mathrm{MSA}_{R P M}$ even for large values of $\sigma_{0}$. Additionally, for a constant surface charge density, the $H N C / M S A_{P M} R D F s$ for counterions and coions fluctuate more strongly and have a steeper slope than the corresponding to $H N C / M S A_{R P M}$, and, consequently, crossings between the profiles for PM and RPM do occur.

For divalent suspensions the RDFs for a $2: 2,0.5 \mathrm{M}$ electrolyte, with $R_{-}=4.25 \AA$ and $R_{+}=8.5 \AA$, and a macrosphere of $D=160 \AA$ are displayed in Fig. 9] for a surface charge $\sigma_{0}=0.08356 \mathrm{C} / \mathrm{m}^{2}$ and in Fig. 10 for $\sigma_{0}=0.3004 \mathrm{C} / \mathrm{m}^{2}$. Again, in the main panels $\mathrm{HNC} / \mathrm{MSA}_{P M}$ and $\mathrm{HNC} / \mathrm{MSA}_{R P M}$ are plotted and in the insets URMGC and MGC. In general, the situation described for the RDFs of monovalent electrolytes is repeated here: URMGC and MGC are monotonic, whereas $\mathrm{HNC} / \mathrm{MSA}_{P M}$ and $\mathrm{HNC} / \mathrm{MSA}_{R P M}$ oscillate, and, for large $\sigma_{0}$, URMGC converge to MGC with no intersections between them and $\mathrm{HNC} / \mathrm{MSA}_{R P M}$ is not the limit of $\mathrm{HNC} / \mathrm{MSA}_{P M}$. Furthermore, Table $\amalg$ ratifies that the counterion contact values, $g_{-}\left(C_{-}\right)$are larger for $\mathrm{HNC} / \mathrm{MSA}_{P M}$ than for $\mathrm{HNC} / \mathrm{MSA}_{R P M}$, and Figs. 9] and 10 evince that the ionic profiles of the former theory wave more intensely than the ones of the latter. Rewardingly, this last pair of structural features of HNC/MSA accords with the ARHNC information for a 2:2 planar EDL obtained by Greberg and Kjellander [66].

As a clear example of how the microscopic structure determines other properties of the EDL, on the grounds of the discussion above we will formulate a 
rationale for the contrasting high- $\sigma_{0}$ behavior of the $\psi_{0}\left(\sigma_{0}\right)$ and $\psi_{I H P}\left(\sigma_{0}\right)$ curves obtained from $\mathrm{PB}$ and HNC/MSA. Aiming for that, let us first condense some of our recent findings in a more convenient form. For size-asymmetric SEDL systems with counterions smaller than coions, if $g_{i}\left(r ; \sigma_{0}\right)$ represents an ionic profile calculated at a given colloidal charge, (i) when $\sigma_{0}$ is varied the URMGC RDFs approach from one side to those of MGC, and the separation between the URMGC and MGC profiles decreases progressively as $\sigma_{0}$ is enlarged, i.e. there are no crossings between the $g_{i}^{U R M G C}\left(r ; \sigma_{0}\right)$ and $g_{i}^{M G C}\left(r ; \sigma_{0}\right)$ functions, and the MGC RDFs bound those of URMGC when $\sigma_{0} \rightarrow \infty$, and (ii) the RDFs at contact for counterions in $\mathrm{HNC} / \mathrm{MSA}_{P M}$ have larger values than in $\mathrm{HNC} / \mathrm{MSA}_{R P M}$, and the ionic densities of $\mathrm{HNC} / \mathrm{MSA}_{P M}$ have a more pronounced slope than the ones of HNC/MSA $R P M$ do, i.e. $g_{i}^{H N C / M S A_{P M}}\left(r ; \sigma_{0}\right)$ and $g_{i}^{H N C / M S A_{R P M}}\left(r ; \sigma_{0}\right)$ present intersections, and, for large $\sigma_{0}$, the RDFs of $H N C / M S A_{P M}$ are not bounded nor have $H N C / M S A_{R P M}$ as a limit. It must be noted that in all the previous statements the comparisons between the theories are performed at fixed $\sigma_{0}$. If we restate explicitely the $\sigma_{0}$-integral, Eq. (28), for a binary electrolyte:

$$
\sigma_{0}=\left(\frac{e \rho_{+} z_{+}}{(D / 2)^{2}}\right) \int_{D / 2}^{\infty}\left[g_{-}(t)-g_{+}(t)\right] t^{2} d t
$$

then, for $\sigma_{0} \geq 0$, this surface charge integral is proportional to the difference between the areas under the $r^{2}$-functions $f_{-}\left(r ; \sigma_{0}\right) \equiv g_{-}\left(r ; \sigma_{0}\right) r^{2}$ and $f_{+}\left(r ; \sigma_{0}\right) \equiv$ $g_{+}\left(r ; \sigma_{0}\right) r^{2}$, for counterions and coions, respectively. In such terms, if we consider the data in each of the Figs. 7 to [10, irrespective of the theory, every of the four pairs of counterion/coion $r^{2}$-functions, $g_{-}\left(r ; \sigma_{0}\right) r^{2}$ and $g_{+}\left(r ; \sigma_{0}\right) r^{2}$, comprises a constant area. This rephrasing of the total electroneutrality condition as a geometrical constraint for the RDFs can now be employed to clarify why the URMGC and MGC potential-charge curves converge for $\sigma_{0} \rightarrow \infty$, whereas the corresponding to $\mathrm{HNC} / \mathrm{MSA}_{P M}$ and $\mathrm{HNC} / \mathrm{MSA}_{R P M}$ do not, or else, why the counterions do not dominate in a description of the size-asymmetric SEDL that surpasses the classical PB equation (for instance, HNC/MSA).

To examine the fulfillment of the constant area restriction as $\sigma_{0}$ is enlarged, in Figs. 11]and12 the $r^{2}$ - functions, $f_{-}\left(r ; \sigma_{0}\right) \equiv g_{-}\left(r ; \sigma_{0}\right) r^{2}$ and $f_{+}\left(r ; \sigma_{0}\right) \equiv g_{+}\left(r ; \sigma_{0}\right) r^{2}$, resulting from the four theories in consideration: URMGC, MGC, HNC/MSA $P M$ and $\mathrm{HNC} / \mathrm{MSA}_{R P M}$, are plotted (in reduced form) for a $2: 2,0.5 \mathrm{M}$ electrolyte of ionic sizes $R_{-}=4.25 \AA$ and $R_{+}=8.5 \AA$, and a macroion of diameter $D=160 \AA$ bearing two different charge densities, $\sigma_{0}=0.08356 \mathrm{C} / \mathrm{m}^{2}$ (in the main sections of the figures) and $\sigma_{0}=0.3004 \mathrm{C} / \mathrm{m}^{2}$ (in the insets). For the URMGC and MGC data of the lower charge, presented in the main panel of Fig. 11] a comparison between the $r^{2}$-functions for coions immediately shows that some area below the URMGC coion function is "lost" in the region $C_{-} \leq r<C_{+}$, owing to the hard-core condition. On the other hard, since the RDFs of URMGC and MGC do not intersect, and remembering the $\mathrm{PB}$ reciprocity property for $n: n$ electrolytes in the region $r \geq C_{+}$:

$$
g_{+}^{P B}(r) g_{-}^{P B}(r)=1,
$$

it is straightforwardly concluded that the ionic profiles $g_{-}^{U R M G C}\left(r ; \sigma_{0}\right)$ and $g_{+}^{U R M G C}\left(r ; \sigma_{0}\right)$ are necessarily located in the region enclosed by $g_{-}^{M G C}\left(r ; \sigma_{0}\right)$ and $g_{+}^{M G C}\left(r ; \sigma_{0}\right)$ (i.e. $\forall r, g_{-}^{U R M G C}\left(r ; \sigma_{0}\right)<g_{-}^{M G C}\left(r ; \sigma_{0}\right)$ and $\left.g_{+}^{U R M G C}\left(r ; \sigma_{0}\right)>g_{+}^{M G C}\left(r ; \sigma_{0}\right)\right)$ as the unique way in which the coion $r^{2}$ - function of URMGC can recover area in $r \geq C_{+}$to assure that the difference functions, $g_{d}\left(r ; \sigma_{0}\right) r^{2}=\left[g_{-}\left(r ; \sigma_{0}\right)-g_{+}\left(r ; \sigma_{0}\right)\right] r^{2}$, of URMGC and MGC preserve a constant area. Additionally, when $\sigma_{0}$ grows (see inset of Fig. 111) the MGC coion RDF at $r=C_{+}$goes down and the coion $r^{2}$-function of URMGC has a minor deficit of area in $C_{-} \leq r<C_{+}$, from which the separation between the URMGC and MGC RDFs diminishes and, eventually, disappears at $\sigma_{0}=\infty$. By contrast, in the HNC/MSA case (Fig. 12) there is also a lost of area in the zone $C_{-} \leq r<C_{+}$for the coion $r^{2}$-function of $\mathrm{HNC} / \mathrm{MSA}_{P M}$, however the possibilty of crossings between the ionic profiles of $\mathrm{HNC} / \mathrm{MSA}_{P M}$ and $\mathrm{HNC} / \mathrm{MSA}_{R P M}$, stemming from the enhancement of the non-monotonicity of the RDFs caused by the size-asymmetry, does not obligate the coincidence of $g_{i}^{H N C / M S A_{P M}}\left(r ; \sigma_{0}\right)$ and $g_{i}^{H N C / M S A_{R P M}}\left(r ; \sigma_{0}\right)$ for large $\sigma_{0}$, even if the difference functions of both theories have the same area. It is from these reasonings that the convergence, for $\sigma_{0} \rightarrow \infty$, between the structural properties of URMGC and MGC and the non-convergence for those of $\mathrm{HNC} / \mathrm{MSA}_{P M}$ and $\mathrm{HNC} / \mathrm{MSA}_{R P M}$ can be understood. A similar analysis done for 1:1 systems (not shown) confirms all the arguments exposed.

Summing up, when the value of $\sigma_{0}$ rises, the difference function $g_{d}\left(r ; \sigma_{0}\right) r^{2}=\left[g_{-}\left(r ; \sigma_{0}\right)-g_{+}\left(r ; \sigma_{0}\right)\right] r^{2}$ for each of the MGC, URMGC, HNC/MSA $R P M$ and $\mathrm{HNC} / \mathrm{MSA}_{P M}$ approximations can accomplish the constraint of a constant area in two ways. On the one hand, when $\sigma_{0} \rightarrow \infty$, the $r^{2}$-integrals (or second moments) of $g_{d}^{U R M G C}\left(r ; \sigma_{0}\right)$ and $g_{d}^{M G C}\left(r ; \sigma_{0}\right)$ remain exactly equal whereas all the rest of integrals of such

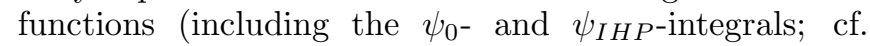
Eqs. (27) and (29) ) become progressively alike, since the RDFs of URMGC and MGC approach asymptotically. On the other, when $\sigma_{0}$ increases, the pair $g_{d}^{H N C / M S A_{P M}}\left(r ; \sigma_{0}\right)$ and $g_{d}^{H N C / M S A_{R P M}}\left(r ; \sigma_{0}\right)$ have the same $r^{2}$-integral but other integrals (such as the $\psi_{0^{-}}$and $\psi_{\left.I H P^{-i n t e g r a l s}\right)}$ become different, since the $\mathrm{HNC} / \mathrm{MSA}_{P M}$ and $\mathrm{HNC} / \mathrm{MSA}_{R P M}$ ionic profiles do not merge. This cogently explains why the $\psi_{0}\left(\sigma_{0}\right)$ and $\psi_{I H P}\left(\sigma_{0}\right)$ curves for URMGC and MGC converge for large $\sigma_{0}$ and those for $\mathrm{HNC} / \mathrm{MSA}_{P M}$ and $\mathrm{HNC} / \mathrm{MSA}_{R P M}$ remain separated. 
To complete this study of the PM-SEDL we switch our attention to the MEP and CRC profiles, $\psi(r)$ and $Q^{*}(r)$, respectively, to gain more insight into the comportment of the diffuse EDL in terms of its neutralization (or charge screening) capacity. In Figs. 13 and 14 the MEP and CRC functions corresponding to the 2:2 data in Figs. 9] and [10] are portrayed, respectively. We have preferred to graph the divalent and not the univalent results since the effects to be discussed have qualitative similarities in the 2:2 and 1:1 systems but are more marked in the former case. In respect to the $\psi(r)$ profiles, the URMGC and MGC MEPs included in the inset of Fig. 13 confirm the monotonic character of the $\mathrm{PB}$ solutions and the convergence between URMGC and MGC for $\sigma_{0} \rightarrow \infty$. In turn, all the HNC/MSA MEP profiles in the main panel of Fig. 13 oscillate and present ample regions of inverted potentials or even show an alternation of signs (see, for example, $\mathrm{HNC} / \mathrm{MSA}_{P M}$ for $\left.\sigma_{0}=0.3004 \mathrm{C} / \mathrm{m}^{2}\right)$. However, the most salient feature in both the $\mathrm{PB}$ and $\mathrm{HNC} / \mathrm{MSA}$ results of $\psi(r)$ is that, in the proximities of the colloid, an SEDL description in which size asymmetry is assumed exhibits lower MEP values compared to the corresponding potentials of the size-symmetric version of the same theory, i.e. $\psi^{H N C / M S A_{P M}}\left(r ; \sigma_{0}\right)<\psi^{H N C / M S A_{R P M}}\left(r ; \sigma_{0}\right)$ and $\psi^{U R M G C}\left(r ; \sigma_{0}\right)<\psi^{M G C}\left(r ; \sigma_{0}\right)$, for $(r-D / 2) \lesssim 7.5 \AA$. A complementary and more quantitative estimation of this can be obtained from the values collected in Table III The observed behavior of the $\psi(r)$ functions close to the surface implies that a size-asymmetric diffuse EDL (either of $\mathrm{HNC} / \mathrm{MSA}_{P M}$ or URMGC) neutralizes more efficiently the native macroion charge than that of a size-symmetric system (of $\mathrm{HNC}_{\mathrm{MSA}} \mathrm{MSM}_{\mathrm{R}}$ or MGC). This generalized dominance of the neutralization power of an unequally-sized electrolyte is remarkable since in the PB approaches there are less counterions close to the macroparticle in URMGC than in MGC, in other words, the counterion contact RDFs comply with $g_{-}^{U R M G C}\left(C_{-} ; \sigma_{0}\right)<g_{-}^{M G C}\left(C_{-} ; \sigma_{0}\right)$, whilst in the $\mathrm{HNC} / \mathrm{MSA}$ theories the contact number of counterions in $\mathrm{HNC} / \mathrm{MSA}_{P M}$ exceeds the prediction of $\mathrm{HNC} / \mathrm{MSA}_{R P M}$, i.e. $g_{-}^{H N C / M S A_{P M}}\left(C_{-} ; \sigma_{0}\right)>$ $g_{-}^{H N C / M S A_{R P M}}\left(C_{-} ; \sigma_{0}\right)$. Such "anomaly" can be justified nicely by the obligated absence of coions in the zone $C_{-} \leq r<C_{+}$for the $\mathrm{HNC} / \mathrm{MSA}_{P M}$ and URMGC theories, which, irrespective of the $\mathrm{PB}$ or $\mathrm{HNC} / \mathrm{MSA}$ approach, allows the counterions to compensate the colloidal charge in a better way than it is done in $\mathrm{HNC} / \mathrm{MSA}_{R P M}$ or MGC, theories where the coions can be indeed present in $C_{-} \leq r<C_{+}$due to the condition $d_{+}=d_{-}$. This, however, does not explain the additional neutralization power of $\mathrm{HNC} / \mathrm{MSA}_{P M}$ over URMGC. For HNC/MSA ${ }_{P M}$ the reason is the increase of the excluded volume, which in turns promotes the coions and counterions adsorption, due to entropic effects, as it has been discussed by Jiménez-Ángeles and LozadaCassou [48]. These last effects also explicate the increase of the counterion adsorption of the $\mathrm{HNC} / \mathrm{MSA}_{P M}$ over
$\mathrm{HNC} / \mathrm{MSA}_{R P M}$ and, consequently, the enhancement of the charge screening in the PM-SEDL.

The behavior of the neutralization capacity detected via the mean electrostatic potential profiles fits very well with the information provided by the cumulative reduced charges. For instance, in the inset of Fig. 14 it can be verified that the $Q^{*}(r)$ functions for URMGC and MGC are monotonic and do not display any change of sign, contrasting with the main panel of the same figure where $\mathrm{HNC} / \mathrm{MSA}_{P M}$ and $\mathrm{HNC} / \mathrm{MSA}_{R P M}$ have CRC curves with oscillations and appreciable charge reversion. Additionally, a genuine and clearer manifestation of the enhanced neutralization capacity of sizeasymmetric models over the symmetrical ones can be gathered directly from the full set of CRC curves in Fig. 14. There, it is visible the faster decay in the cumulative charges corresponding to $\mathrm{HNC} / \mathrm{MSA}_{P M}$ and $\mathrm{UR}$ $\mathrm{MGC}$ with respect to those of $\mathrm{HNC} / \mathrm{MSA}_{R P M}$ and MGC, i.e. $Q^{* H N C / M S A_{P M}}\left(r ; \sigma_{0}\right)<Q^{* H N C / M S A_{R P M}}\left(r ; \sigma_{0}\right)$ and $Q^{* U R M G C}\left(r ; \sigma_{0}\right)<Q^{* M G C}\left(r ; \sigma_{0}\right)$, for $(r-D / 2) \lesssim$ $10 \AA$.

Notably, for a given $\sigma_{0}$, among all the reviewed theories, the $\mathrm{HNC} / \mathrm{MSA}_{P M}$ formalism is always associated to the strongest fluctuations and to the more accentuated sign inversions in $\psi(r)$ and to the largest neutralization and charge reversal effects in $Q^{*}(r)$. This fact, together with the rest of findings revealed by the analysis of the $\psi(r)$ and $Q^{*}(r)$ profiles, conforms an extra and compelling testimony to the relevance of using sizeasymmetric models when a more faithful description of colloidal suspensions is sought.

To end, we want to point out that, for our $n: n$ systems, at the distances where the RDFs show a charge inversion the CRCs go through a minimum or maximum, as it was noted previously by Wang et al. in the RPMEDL for cylindrical geometry [33]. This signifies that, for a valence-symmetric RPM or PM electrolyte, charge inversion implies charge reversal. What is more, from basic electrostatics for the SEDL, it is possible to prove that $\frac{d \psi(r)}{d r}=-\frac{Q(r)}{\epsilon r^{2}}$ (similar equations for other geometries are given in Refs. [33, 48]), thus, the distances at which the $\mathrm{CRC}$ is zero correspond precisely to those where the mean electrostatic potential presents extremum values, whence, the existence of oscillations in $Q^{*}(r)$ should not be enough to produce fluctuations in $\psi(r)$, i.e. a change of sign in the CRC is indeed required.

\section{CONCLUSIONS}

In this work a survey of the size-asymmetric spherical electrical double layer in the primitive model was performed by using the $\mathrm{HNC} / \mathrm{MSA}$ integral equation. After the correctness of the HNC/MSA description was attested by comparing our numerical results with simulations and previous ARHNC data (in the planar limit), we have carried out a study of the structural and chargepotential relationship for archetypal cases of $1: 1$ and 2:2 
systems with a size-asymmetry ratio of 2 . To assess the importance of the size-asymmetry effects, we collated our predictions with those of $\mathrm{HNC} / \mathrm{MSA}$ in the RPM, and also with the corresponding to the classical Poisson-Boltzmann approaches: URMGC and MGC, to exhibit the notable quantitative and qualitative discrepances existing between punctual and non-punctual EDL theories. The main conclusion of this paper is that sizeasymmetry is an essential improvement to consider in studies of the SEDL since heightens ionic size correlation effects (e.g. nonmonotonicity, charge inversion and charge reversal) already seen in the RPM case and, consequently, unveils new and interesting phenomenology absent in size-symmetric systems. As a conspicuous example of these novel effects, it is evidenced that counterions do not always dominate in the PM-SEDL, as it is suggested by the PB point of view, and the wherefores of this fact are traced back to the detailed characteristics of the EDL structure. In particular, the contrasting high- $\sigma_{0}$ behavior displayed by the $\mathrm{PB}$ and $\mathrm{HNC} / \mathrm{MSA}$ potentialcharge relationships, i.e. the convergence, for $\sigma_{0} \rightarrow \infty$, between the URMGC and MGC $\psi_{0}\left(\sigma_{0}\right)$ and $\psi_{I H P}\left(\sigma_{0}\right)$ curves and the non-merging between the $\mathrm{HNC} / \mathrm{MSA}_{P M}$ and $\mathrm{HNC} / \mathrm{MSA}_{R P M}$ ones, was elucidated by the following rational: for a system with fixed parameters, when $\sigma_{0}$ increases the RDFs of URMGC and $M G C$, and all their integrals and associated EDL properties (e.g. $\psi_{0}$ and $\left.\psi_{I H P}\right)$, go similar, whereas for $H N C / M S A_{P M}$ and $H N C / M S A_{R P M}$, even if their RDFs can have the same second moment, in general, their corresponding shapes and ensuing properties $\left(\psi_{0}\right.$ and/or $\left.\psi_{I H P}\right)$ could be completely different.

In forthcoming publications some other appealing phenomena occurring in PM-SEDL, as overcharging 48] and the advent of anomalous differential capacities [92], will be addressed by means of our integral equation approach.

\section{Acknowledgments}

E. G.-T. thanks the support by CONACYT (NC0072) and PROMEP.
[1] F. Fenell-Evans and H. Wennerström, The Colloidal Domain: Where Physics, Chemistry, Biology and Technology Meet (Wiley-VCH, New York, 1994).

[2] P. C. Hiemenz and R. Rajagopalan, Principles of Colloid and Surface Chemistry (Marcel Dekker, New York, 1997).

[3] S. S. Dukhin and B. V. Derjaguin, Surface and Colloid Science (E. Matijevic, Ed.), Vol. 7, Chaps. 1 and 2 (Wiley, New York, 1974).

[4] R. J. Hunter, Zeta Potential in Colloid Science (Academic Press, New York, 1981).

[5] R. D. Vold and M. J. Vold, Colloid and Interface Chemistry (Addison-Wesley, Reading, MA, 1983).

[6] R. J. Hunter, Foundations of Colloid Science (Clarendon, Oxford, 1987).

[7] W. B. Russell, D. A. Saville, and W. R. Schowalter, Colloidal Dispersions (Cambridge University Press, Cambridge, UK, 1989).

[8] M. Elimelech and C. R. O'Melia, Environ. Sci. Technol. 24, 1528 (1990).

[9] S. L. Carnie, D. Y. C. Chan, D. J. Mitchell, and B. W. Ninham, J. Chem. Phys. 74, 1472 (1981).

[10] S. L. Carnie and G. M. Torrie, Adv. Chem. Phys. 56, 141 (1984).

[11] P. Attard, Adv. Chem. Phys. 92, 1 (1996).

[12] M. Lozada-Cassou, R. Saavedra-Barrera, and D. Henderson, J. Chem. Phys. 77, 5150 (1982).

[13] E. Gonzales-Tovar, M. Lozada-Cassou, and D. Henderson, J. Chem. Phys. 83, 361 (1985); 87, 5581 (1987).

[14] V. Vlachy and D. A. McQuarrie, J. Chem. Phys. 83, 1927 (1985).

[15] V. Vlachy and D. A. McQuarrie, J. Phys. Chem. 90, 3248 (1986).

[16] E. González-Tovar and M. Lozada-Cassou, J. Phys. Chem. 93, 3761 (1989).
[17] M. Lozada-Cassou and E. Díaz-Herrera, J. Chem. Phys. 92, 1194 (1990).

[18] L. Yeomans, S. E. Feller, E. Sánchez, and M. LozadaCassou, J. Chem. Phys. 98, 1436 (1993).

[19] J. Yu, L. Degrève, and M. Lozada-Cassou, Phys. Rev. Lett. 79, 3656 (1997).

[20] R. Kjellander and S. Marčelja, Chem. Phys. Lett. 127 402 (1986).

[21] R. Kjellander, J. Chem. Phys. 88, 7129 (1988); 89, 7649 (1988).

[22] M. Plischke and D. Henderson, J. Chem. Phys. 88, 2712 (1988).

[23] H. Greberg, R. Kjellander, and T. Akesson, Mol. Phys. 92, 35 (1997).

[24] L. Mier-y-Terán, S. H. Suh, H. S. White, and H. T. Davis, J. Chem. Phys. 92, 5087 (1990).

[25] E. Kierlik and M. L. Rosinberg, Phys. Rev. A 44, 5025 (1991).

[26] C. N. Patra and S. K. Gosh, J. Chem. Phys. 100, 5219 (1994).

[27] C. N. Patra and S. K. Gosh, J. Chem. Phys. 101, 4143 (1994).

[28] D. Boda, W. R. Fawcett, D. Henderson, and S. Sokolowski, J. Chem. Phys. 116, 7170 (2002).

[29] C. N. Patra and S. K. Gosh, J. Chem. Phys. 117, 8938 (2002).

[30] D. Boda, D. Henderson, L. Mier-y-Terán, and S. Sokolowski, J. Phys.: Condens. Matter 14, 11945 (2002).

[31] D. Boda, D. Henderson, P. Plaschko, and R. Fawcett, Mol. Simul. 30, 137 (2004).

[32] Y. X. Yu, J. Z. Wu, and G. H. Gao, J. Chem. Phys. 120, 7223 (2004).

[33] K. Wang, Y. X. Yu, and G. H. Gao, Phys. Rev. E 70, $011912(2004)$.

[34] C. W. Outhwaite and L. B. Bhuiyan, J. Chem. Soc. Fara- 
day Trans. 2 79, 707 (1983)

[35] C. W. Outhwaite and L. B. Bhuiyan, Electrochim. Acta 36, 1747 (1991).

[36] C. W. Outhwaite and L. B. Bhuiyan, Mol. Phys. 74, 367 (1991).

[37] L. B. Bhuiyan and C. W. Outhwaite, Philos. Mag. B 69, 1051 (1994).

[38] G. M. Torrie and J. P. Valleau, J. Chem. Phys. 73, 5807 (1980).

[39] G. M. Torrie and J. P. Valleau, J. Phys. Chem. 86, 3251 (1982).

[40] L. Degrève, M. Lozada-Cassou, E. Sánchez, and E. González-Tovar, J. Chem. Phys. 98, 8905 (1993).

[41] L. Degrève and M. Lozada-Cassou, Mol. Phys. 86, 759 (1995).

[42] T. Terao and T. Nakayama, Phys. Rev. E 63, 041401 (2001).

[43] M. Le Bret and B. H. Zimm, Biopolymers 23, 271 (1984).

[44] V. Vlachy and A. D. J. Haymet, J. Chem. Phys. 84, 5874 (1986).

[45] J. C. Gil Montoro and J. L. F. Abascal, J. Chem. Phys. 103, 8273 (1995).

[46] H. Ni, C. F. Anderson, and M. T. Record Jr., J. Phys. Chem. B 103, 3489 (1999).

[47] M. Deserno, F. Jiménez-Ángeles, C. Holm, and M. Lozada-Cassou, J. Phys. Chem. B 105, 10983 (2001).

[48] F. Jiménez-Ángeles and M. Lozada-Cassou, J. Phys. Chem. B 108, 7286 (2004).

[49] M. Lozada-Cassou, E. González-Tovar, and W. Olivares, Phys. Rev. E 60, R17 (1999).

[50] M. Lozada-Cassou and E. González-Tovar, J. Colloid Interface Sci. 239, 285 (2001); 240, 644 (2001).

[51] M. Quesada-Pérez, E. González-Tovar, A. MartínMolina, M.Lozada-Cassou, and R. Hidalgo-Álvarez, Chemphyschem 4, 234 (2003).

[52] M. Quesada-Pérez, E. González-Tovar, A. MartínMolina, M. Lozada-Cassou, and R. Hidalgo-Álvarez, Colloids and Surfaces A, submitted.

[53] T. Das, D. Bratko, L. B. Bhuiyan, and C. W. Outhwaite, J. Chem. Phys. 107, 9197 (1997).

[54] V. Vlachy, Annu. Rev. Phys. Chem. 50, 145 (1999).

[55] J. G. Kirkwood, J. Chem. Phys. 2, 767 (1934).

[56] R. H. Fowler, Statistical Mechanics (Cambridge University Press, London, 1966).

[57] P. M. V. Résibois, Electrolyte Theory: An Elementary Introduction to a Microscopic Approach (Harper \& Row, New York, 1968).

[58] D. A. McQuarrie, Statistical Mechanics (Harper Collins Publishers, New York, 1976).

[59] G. M. Torrie and J. P. Valleau, Chem. Phys. Lett. 65, 343 (1979).

[60] J. P. Valleau and G. M. Torrie, J. Chem. Phys. 76, 4623 (1982).

[61] L. B. Bhuiyan, L. Blum, and D. Henderson, J. Chem. Phys. 78, 442 (1983).

[62] J. J. Spitzer, J. Colloid Interface Sci. 92, 198 (1983).

[63] A. F. Khater, D. Henderson, L. Blum, and L. B. Bhuiyan, J. Phys. Chem. 88, 3682 (1984).

[64] U. Marini Bettolo Marconi, J. Wiechen, and F. Forstmann, Chem. Phys. Lett. 107, 609 (1984).

[65] C. W. Outhwaite and L. B. Bhuiyan, J. Chem. Phys. 84, 3461 (1986).

[66] H. Greberg and R. Kjellander, J. Chem. Phys. 108, 2940
(1998).

[67] M. Valiskó, D. Henderson, and D. Boda, J. Phys. Chem. $\mathrm{B}$, in press.

[68] M. Lozada-Cassou and F. Jiménez-Ángeles, in Coloides e Interfases, edited by L. M. Liz Marzán et al. (Universidad de Vigo, Vigo, Spain, 2003).

[69] F. Jiménez-Ángeles and M. Lozada-Cassou, J. Phys. Chem. B 108, 1719 (2004).

[70] In Ref. 63] the MSA expression for the potential difference across a PM-SEDL was obtained, however, such formula has only been used in the planar limit in Ref. 67].

[71] L. B. Bhuiyan and C. W. Outhwaite, Phys. Chem. Chem. Phys. 6, 3467 (2004).

[72] M. Lozada-Cassou, J. Chem. Phys. 75, 1412 (1981).

[73] M. Lozada-Cassou, J. Chem. Phys. 77, 5258 (1982).

[74] M. Lozada-Cassou, in Fundamentals of Inhomogeneous Fluids, edited by D. Henderson (Marcel Dekker, New York, 1992).

[75] J. Lyklema and J. Th. G. Overbeek, J. Colloid Sci. 16, 501 (1961).

[76] D. Stigter, J. Phys. Chem. 68, 3600 (1964).

[77] J. Th. G. Overbeek and P. H. Wiersema, in Electrophoresis, edited by M. Bier (Academic Press, New York, 1967), Vol. II.

[78] J. Lyklema, J. Colloid Interface Sci. 58, 242 (1977).

[79] S. G. Brush, H. L. Sahlin, and E. Teller, J. Chem. Phys. 45, 2102 (1966).

[80] D. N. Card and J. P. Valleau, J. Chem. Phys. 52, 6232 (1970).

[81] J. P. Valleau and L. K. Cohen, J. Chem. Phys. 72, 5935 (1980).

[82] S. H. Rodge and J. Hafskjold, Mol. Phys. 48, 1241 (1983).

[83] M. P. Allen and D. J. Tildesley Computer Simulation of Liquids (Oxford University Press, New York, 1989).

[84] D. Frenkel and B. Smit Understanding Molecular Simulation (Academic Press, London, 2002).

[85] M. Tckerman, B. J. Berne, and G. J. Martyna, J. Chem. Phys. 97, 1990 (1992).

[86] M. Lozada-Cassou and D. Henderson, J. Phys. Chem. 87, 2821 (1983).

[87] P. Attard, D. Wei, and G. N. Patey, J. Chem. Phys. 96, 3767 (1992).

[88] G. M. Torrie, J. Chem. Phys. 96, 3772 (1992).

[89] M. B. Partenskii and P. C. Jordan, J. Chem. Phys. 99, 2992 (1993).

[90] M. J. Booth, A. C. Eaton, and A. D. J. Haymet, J. Chem. Phys. 103, 417 (1995).

[91] A. C. Eaton and A. D. J. Haymet, J. Chem. Phys. 114, 10938 (2001).

[92] E. González-Tovar, F. Jiménez-Ángeles, R. Messina, and M. Lozada-Cassou, J. Chem. Phys. 120, 9782 (2004).

[93] G. M. Torrie, J. P. Valleau, and C. W. Outhwaite, J. Chem. Phys. 81, 6296 (1984).

[94] L. Blum, Mol. Phys. 30, 1529 (1975).

[95] L. Blum and J. S. Høye, J. Phys. Chem. 81, 1311 (1977).

[96] K. Hiroike, Mol. Phys. 33, 1195 (1977).

[97] R. J. Baxter, J. Chem. Phys. 52, 4559 (1970).

[98] J. L. Lebowitz, Phys. Rev. 133, A895 (1964). 


\section{APPENDIX A: EXPLICIT MSA BULK DIRECT CORRELATION FUNCTIONS}

The direct correlation functions of a PM bulk electrolyte in the mean spherical approximation have been obtained analytically by Blum 94, 95 and Hiroike 96 for the general case of $n$ species with arbitrary size and charge. The only restriction is the electroneutrality condition:

$$
\sum_{i=1}^{n} \rho_{i} z_{i}=0 .
$$

The MSA direct correlation functions can be expressed as:

$$
c_{i j}(s)=c_{i j}^{h s}(s)+c_{i j}^{e l e c}(s),
$$

where $c_{i j}^{h s}(s)$ is the hard-sphere contribution and $c_{i j}^{\text {elec }}(s)$ is the electrostatic part.

\section{Electrostatic contribution}

Following the Baxter method 97] Blum found that the excess properties of an electrolyte can be written in terms of the parameter $\Gamma$. When a binary mixture of hard charged spheres of diameters $R_{1}$ and $R_{2}$ and valences $z_{1}$ and $z_{2}$ is considered, this parameter can be obtained solving the trascendental algebraic equation (it is assumed for definiteness that $R_{2} \geq R_{1}$ ):

$$
\Gamma^{2}=\pi \omega D(\Gamma)
$$

where

$$
\begin{gathered}
D(\Gamma)=\rho_{1} X_{1}^{2}(\Gamma)+\rho_{2} X_{2}^{2}(\Gamma), \\
X_{1}(\Gamma)=\frac{z_{1}}{1+\Gamma R_{1}}+\frac{R_{1}^{2} \chi(\Gamma)}{1+\Gamma R_{1}}, \\
X_{2}(\Gamma)=\frac{z_{2}}{1+\Gamma R_{2}}+\frac{R_{2}^{2} \chi(\Gamma)}{1+\Gamma R_{2}}, \\
\chi=\frac{-c\left\{\rho_{1} R_{1} z_{1}\left(1+\Gamma R_{1}\right)^{-1}+\rho_{2} R_{2} z_{2}\left(1+\Gamma R_{2}\right)^{-1}\right\}}{1+c\left\{\rho_{1} R_{1}^{3}\left(1+\Gamma R_{1}\right)^{-1}+\rho_{2} R_{2}^{3}\left(1+\Gamma R_{2}\right)^{-1}\right\}}(\mathrm{A} 7) \\
\Upsilon=\left(1+\Gamma R_{1}\right)\left(1+\Gamma R_{2}\right),
\end{gathered}
$$

$$
c=\frac{\pi}{2}\left[1-\frac{\pi}{6}\left(\rho_{1} R_{1}^{3}+\rho_{2} R_{2}^{3}\right)\right]^{-1}
$$

and

$$
\omega=\frac{e^{2}}{\epsilon k_{B} T},
$$

with $\rho_{1}$ and $\rho_{2}$ being the numerical densities of the two species.

If we define

$$
\lambda=\frac{R_{2}-R_{1}}{2}
$$

$$
R_{12}=\frac{R_{1}+R_{2}}{2}
$$

and

$$
P_{i j}=\frac{z_{i} z_{j} e^{2}}{\epsilon k_{B} T}
$$

then $c_{i j}^{\text {elec }}(s)(i, j=1,2)$ is given explicitly by

$$
c_{12}^{\text {elec }}(s)=\beta_{0}, 0 \leq s \leq \lambda
$$

$$
c_{12}^{\text {elec }}(s)=\alpha_{0} s^{-1}+\alpha_{1}+\alpha_{2} s+\alpha_{3} s^{3}, \lambda \leq s<R_{12},
$$

$$
c_{12}^{\text {elec }}(s)=-P_{12} s^{-1}, s>R_{12},
$$

where

$$
\begin{gathered}
\beta_{0}=2 \omega\left[\left(\frac{\Gamma R_{1}^{4}-2 R_{1}^{3}}{3\left(1+\Gamma R_{1}\right)}\right) \chi^{2}+\right. \\
\left.\left(\frac{z_{1}\left(R_{2}-R_{1}\right)}{\Upsilon}\right) \chi-\left(\frac{z_{1} z_{2} \Gamma}{1+\Gamma R_{2}}\right)\right], \quad(\mathrm{A} 1 \\
\alpha_{0}=\frac{\omega\left(R_{2}-R_{1}\right)^{2}}{16 \Upsilon}\left(\left[4\left(R_{1}^{2}+R_{2}^{2}\right)-4 \Gamma^{2} R_{1}^{2} R_{2}^{2}\right.\right. \\
\left.\left.-\left(R_{1}-R_{2}\right)^{2} \Upsilon\right] \chi^{2}+\left[4\left(z_{1}+z_{2}\right)\right] \chi+4 z_{1} z_{2} \Gamma^{2}\right),
\end{gathered}
$$


where

$$
\begin{aligned}
\alpha_{1} & =\frac{\omega}{\Upsilon}\left(\left[z_{1}\left(R_{2}-R_{1}\right)+z_{2}\left(R_{1}-R_{2}\right)\right] \chi-2 \Gamma z_{1} z_{2}\right. \\
& -\left(R_{1}+R_{2}\right) z_{1} z_{2} \Gamma^{2}+\left[\frac{R_{1}^{3}}{3}\left(1+\Gamma R_{2}\right)\left(\Gamma R_{1}-2\right)\right. \\
+ & \left.\left.\frac{R_{2}^{3}}{3}\left(1+\Gamma R_{1}\right)\left(\Gamma R_{2}-2\right)\right] \chi^{2}\right), \\
\alpha_{2} & =\frac{\omega}{\Upsilon}\left(\left[z_{1}+z_{2}\right] \chi+\Gamma^{2} z_{1} z_{2}+\right. \\
& {\left.\left[R_{1}^{2}+R_{2}^{2}-\Gamma^{2} R_{1}^{2} R_{2}^{2}-\frac{\left(R_{1}-R_{2}\right)^{2} \Upsilon}{2}\right] \chi^{2}\right) }
\end{aligned}
$$

and

$$
\begin{gathered}
\alpha_{3}=\frac{w \chi^{2}}{3} . \\
c_{11}^{\text {elec }}(s)=i_{0}+i_{1} s+i_{2} s^{3}, 0 \leq s \leq R_{1}, \\
c_{11}^{\text {elec }}(s)=-P_{11} s^{-1}, s>R_{1}
\end{gathered}
$$

with

$$
\begin{gathered}
i_{0}=\frac{\omega}{\left(1+\Gamma R_{1}\right)^{2}}\left(-2 \Gamma z_{1}^{2}-2 R_{1} z_{1}^{2} \Gamma^{2}+\right. \\
\left.\left[\frac{2}{3} R_{1}^{3}\left(\Gamma R_{1}-2\right)\left(1+\Gamma R_{1}\right)\right] \chi^{2}\right), \quad(\mathrm{A} 24) \\
i_{1}=\frac{\omega}{\left(1+\Gamma R_{1}\right)^{2}}\left(\left[2 R_{1}^{2}-\left(\Gamma R_{1}^{2}\right)^{2}\right] \chi^{2}\right. \\
\left.+\left[2 z_{1}\right] \chi+\Gamma^{2} z_{1}^{2}\right)
\end{gathered}
$$

and

$$
i_{2}=\frac{w \chi^{2}}{3}
$$

Finally,

$$
\begin{gathered}
c_{22}^{\text {elec }}(s)=j_{0}+j_{1} s+j_{2} s^{3}, 0 \leq s \leq R_{2}, \\
c_{22}^{\text {elec }}(s)=-P_{22} s^{-1}, s>R_{2},
\end{gathered}
$$

$$
\begin{gathered}
j_{0}=\frac{\omega}{\left(1+\Gamma R_{2}\right)^{2}}\left(-2 \Gamma z_{2}^{2}-2 R_{2} z_{2}^{2} \Gamma^{2}+\right. \\
\left.\left[\frac{2}{3} R_{2}^{3}\left(\Gamma R_{2}-2\right)\left(1+\Gamma R_{2}\right)\right] \chi^{2}\right),
\end{gathered}
$$

$$
\begin{gathered}
j_{1}=\frac{\omega}{\left(1+\Gamma R_{2}\right)^{2}}\left(\left[2 z_{2}\right] \chi+\Gamma^{2} z_{2}^{2}+\right. \\
\left.\left[2 R_{2}^{2}-\left(\Gamma R_{2}^{2}\right)^{2}\right] \chi^{2}\right)
\end{gathered}
$$

and

$$
j_{2}=\frac{w \chi^{2}}{3}
$$

\section{Hard sphere contribution}

The exact solution for the Percus-Yevick equation of a hard spheres mixture with $n$ species was analyticaly obtained by Lebowitz 98]. Following the notation of Lebowitz, we define for a binary mixture

$$
\begin{gathered}
\xi=\frac{\pi}{6}\left(\rho_{1} R_{1}^{3}+\rho_{2} R_{2}^{3}\right), \\
a_{1}=(1-\xi)^{-3}\left\{1+\xi+\xi^{2}+\frac{\pi}{6} R_{1}^{3}\left(\rho_{1}+\rho_{2}\right)(1+2 \xi)\right. \\
-\frac{\pi}{2}\left(R_{2}-R_{1}\right)^{2} \rho_{2}\left[\frac{\pi}{6} R_{2} \rho_{1} R_{1}^{3}+\left(R_{1}+R_{2}\right)+\right. \\
\left.\left.\frac{\pi}{6} R_{1} R_{2}\left(\rho_{1} R_{1}^{2}+\rho_{2} R_{2}^{2}\right)\right]\right\}+\frac{\pi}{2} R_{1}^{3}(1-\xi)^{-4}\{ \\
\left(\rho_{1}+\rho_{2}\right)\left[1+\xi+\xi^{2}\right]-\frac{\pi}{2} \rho_{1} \rho_{2}\left(R_{2}-R_{1}\right)^{2}[ \\
\left.\left.\left(R_{1}+R_{2}\right)+\frac{\pi}{6} R_{1} R_{2}\left(\rho_{1} R_{1}^{2}+\rho_{2} R_{2}^{2}\right)\right]\right\}, \\
\left.\left.\frac{\pi}{6} R_{1} R_{2}\left(\rho_{1} R_{1}^{2}+\rho_{2} R_{2}^{2}\right)\right]\right\}+\frac{\pi}{2} R_{2}^{3}(1-\xi)^{-4}\{ \\
a_{2}=(1-\xi)^{-3}\left\{1+\xi+\xi^{2}+\frac{\pi}{6} R_{2}^{3}\left(\rho_{1}+\rho_{2}\right)(1+2 \xi)\right. \\
-\frac{\pi}{2}\left(R_{2}-R_{1}\right)^{2} \rho_{1}\left[\frac{\pi}{6} R_{1} \rho_{2} R_{2}^{3}+\left(R_{1}+R_{2}\right)+\right.
\end{gathered}
$$




$$
\begin{aligned}
& \left(\rho_{1}+\rho_{2}\right)\left[1+\xi+\xi^{2}\right]-\frac{\pi}{2} \rho_{1} \rho_{2}\left(R_{2}-R_{1}\right)^{2}[ \\
& \left.\left.\left(R_{1}+R_{2}\right)+\frac{\pi}{6} R_{1} R_{2}\left(\rho_{1} R_{1}^{2}+\rho_{2} R_{2}^{2}\right)\right]\right\} \\
& d=\frac{\pi}{12}\left[\rho_{1} a_{1}+\rho_{2} a_{2}\right] \\
& g_{11}\left(R_{1}\right)=\left\{1+\frac{\xi}{2}+\frac{\pi}{4} \rho_{2} R_{2}^{2}\left(R_{1}-R_{2}\right)\right\}(1-\xi)^{-2} \\
& g_{22}\left(R_{2}\right)=\left\{1+\frac{\xi}{2}+\frac{\pi}{4} \rho_{1} R_{1}^{2}\left(R_{2}-R_{1}\right)\right\}(1-\xi)^{-2} \\
& g_{12}\left(R_{12}\right)=\left[R_{2} g_{11}\left(R_{1}\right)+R_{1} g_{22}\left(R_{2}\right)\right]\left(R_{1}+R_{2}\right)^{-1} \\
& b_{1}=-\frac{\pi}{4}\left[4 \rho_{1} R_{1}^{2} g_{11}^{2}\left(R_{1}\right)+\rho_{2}\left(R_{1}+R_{2}\right)^{2} g_{12}^{2}\left(R_{12}\right)\right] \text {, } \\
& b_{2}=-\frac{\pi}{4}\left[4 \rho_{2} R_{2}^{2} g_{22}^{2}\left(R_{2}\right)+\rho_{1}\left(R_{1}+R_{2}\right)^{2} g_{12}{ }^{2}\left(R_{12}\right)\right]
\end{aligned}
$$

and

$$
\begin{aligned}
b= & -\frac{\pi}{2}\left[\rho_{1} R_{1} g_{11}\left(R_{1}\right)+\right. \\
& \left.\rho_{2} R_{2} g_{22}\left(R_{2}\right)\right]\left(R_{1}+R_{2}\right) g_{12}\left(R_{12}\right) .
\end{aligned}
$$

The $c_{i j}^{h s}(s)(i, j=1,2)$ are then given by

$$
\begin{gathered}
c_{i i}^{h s}(s)=-a_{i}-b_{i} s-d s^{3}, s \leq R_{i}, i=1,2, \\
c_{i i}^{h s}(s)=0, s>R_{i}, i=1,2, \\
c_{12}^{h s}(s)=c_{21}^{h s}(s)=-a_{1}, 0 \leq s \leq \lambda, \\
c_{12}^{h s}(s)=c_{21}^{h s}(s)=-a_{1}-\gamma_{0} s^{-1} \\
-\gamma_{1}-\gamma_{2} s-d s^{3}, \lambda \leq s \leq R_{12}, \\
c_{12}^{h s}(s)=c_{21}^{h s}(s)=0, s>R_{12},
\end{gathered}
$$

where

$$
\begin{gathered}
\gamma_{0}=\lambda^{2}\left(b-3 d \lambda^{2}\right) \\
\gamma_{1}=8 \lambda^{3} d-2 \lambda b
\end{gathered}
$$

and

$$
\gamma_{2}=b-6 \lambda^{2} d
$$

Finally, if we define the constants

$$
A_{1}=i_{0}-a_{1} ; \quad A_{2}=i_{1}-b_{1} ; \quad A_{3}=i_{2}-d
$$

$$
B_{1}=j_{0}-a_{2} ; \quad B_{2}=j_{1}-b_{2} ; \quad B_{3}=j_{2}-d
$$

$$
E_{1}=\beta_{0}-a_{1} ; \quad F_{1}=\alpha_{0}-\gamma_{0}
$$

$$
F_{2}=\alpha_{1}-a_{1}-\gamma_{1} ; \quad F_{3}=\alpha_{2}-\gamma_{2} ; \quad F_{4}=\alpha_{3}-d ;
$$
we can write the bulk correlation functions as

$$
c_{i j}(s)=c_{i j}^{h s}(s)+c_{i j}^{e l e c}(s)=c_{i j}^{i n t}(s)+c_{i j}^{e x t}(s),
$$

where

$$
c_{11}^{i n t}(s)=P_{11} s^{-1}+A_{1}+A_{2} s+A_{3} s^{3}, 0 \leq s \leq R_{1},
$$

$$
c_{11}^{i n t}(s)=0, s>R_{1}
$$

$c_{22}^{i n t}(s)=P_{22} s^{-1}+B_{1}+B_{2} s+B_{3} s^{3}, 0 \leq s \leq R_{2}$,

$$
c_{22}^{i n t}(s)=0, s>R_{2}
$$

$$
c_{12}^{i n t}(s)=c_{21}^{i n t}(s)=P_{12} s^{-1}+E_{1}, 0 \leq s \leq \lambda
$$

$$
\begin{aligned}
& c_{12}^{i n t}(s)=c_{21}^{i n t}(s)=P_{12} s^{-1}+ \\
& F_{1} s^{-1}+F_{2}+F_{3} s+F_{4} s^{3}, \lambda \leq s \leq R_{12}
\end{aligned}
$$




$$
\begin{gathered}
c_{12}^{i n t}(s)=c_{21}^{i n t}(s)=0, s \geq R_{12} \\
c_{11}^{e x t}(s)=-P_{11} s^{-1}, s>0 \\
c_{22}^{e x t}(s)=-P_{22} s^{-1}, s>0
\end{gathered}
$$

and

$$
c_{12}^{e x t}(s)=c_{21}^{e x t}(s)=-P_{12} s^{-1}, s>0
$$

\section{APPENDIX B: EXPLICIT FORM OF THE $I_{i}(r)$ AND $H_{i}(r)$ TERMS IN THE HNC/MSA INTEGRAL EQUATIONS FOR THE PM-SEDL}

Due to the radial symmetry of the system, the angular dependence $(\theta, \phi)$ of the equations (11)-(12), (16)-19) and (21)-(24) can be resolved since the angular integrals can be performed analytically.

The final form of the $I_{i}(r)$ and $H_{i}(r)$ terms can be given if we define

$$
J\left(x_{f}, l, r, t\right)=\frac{1}{(l+2) r t}\left[x_{f}^{l+2}-|r-t|^{l+2}\right],
$$

and

$$
J^{\prime}\left(x_{i}, x_{f}, l, r, t\right)=\frac{1}{(l+2) r t}\left[x_{f}^{l+2}-x_{i}^{l+2}\right] .
$$

Thus, if

$$
\frac{D}{2}+\frac{R_{1}}{2} \leq r \leq \frac{D}{2}+\frac{3}{2} R_{1}
$$

we have

$$
I_{1}(r)=\int_{\frac{D}{2}+\frac{R_{1}}{2}}^{r+R_{1}} K I_{1} a(r, t) d t+\int_{\frac{D}{2}+\frac{R_{1}}{2}}^{\infty} K I_{1} b(r, t) d t
$$

and, if

$$
\frac{D}{2}+\frac{3}{2} R_{1}<r<\infty
$$

then

$$
I_{1}(r)=\int_{r-R_{1}}^{r+R_{1}} K I_{1} a(r, t) d t+\int_{\frac{D}{2}+\frac{R_{1}}{2}}^{\infty} K I_{1} b(r, t) d t
$$

where

$$
\begin{aligned}
& K I_{1} a(r, t)=2 \pi g_{1}(t) t^{2}\left(P_{11} J\left(R_{1},-1, r, t\right)+\right. \\
& \quad A_{1} J\left(R_{1}, 0, r, t\right)+A_{2} J\left(R_{1}, 1, r, t\right)+ \\
& \left.\quad A_{3} J\left(R_{1}, 3, r, t\right)\right)
\end{aligned}
$$

and

$$
K I_{1} b(r, t)=-2 \pi P_{11} g_{1}(t) \frac{t}{r}(r+t-|r-t|)
$$

If

$$
\frac{D}{2}+\frac{R_{1}}{2} \leq r<\infty
$$

then

$$
I_{2}(r)=4 \pi\left(\frac{P_{11} R_{1}^{2}}{2}+\frac{A_{1} R_{1}^{3}}{3}+\frac{A_{2} R_{1}^{4}}{4}+\frac{A_{3} R_{1}^{6}}{6}\right)
$$

If

$$
\frac{D}{2}+\frac{R_{1}}{2} \leq r \leq \frac{D}{2}+\frac{R_{2}}{2}+\lambda
$$

then

$$
\begin{aligned}
& I_{3}(r)=\int_{\frac{D}{2}+\frac{R_{2}}{2}}^{r+\lambda} K I_{3} a(r, t) d t+ \\
& \int_{r+\lambda}^{r+R_{12}} K I_{3} b(r, t) d t+\int_{\frac{D}{2}+\frac{R_{2}}{2}}^{\infty} K I_{3} c(r, t) d t,
\end{aligned}
$$

if

$$
\frac{D}{2}+\frac{R_{2}}{2}+\lambda<r<\frac{D}{2}+\frac{R_{2}}{2}+R_{12},
$$

then

$$
\begin{array}{r}
I_{3}(r)=\int_{\frac{D}{2}+\frac{R_{2}}{2}}^{r-\lambda} K I_{3} b(r, t) d t+\int_{r-\lambda}^{r+\lambda} K I_{3} a(r, t) d t \\
+\int_{r+\lambda}^{r+R_{12}} K I_{3} b(r, t) d t+\int_{\frac{D}{2}+\frac{R_{2}}{2}}^{\infty} K I_{3} c(r, t) d t
\end{array}
$$

and, if 


$$
\frac{D}{2}+\frac{R_{2}}{2}+R_{12} \leq r<\infty
$$

then

$$
\begin{array}{r}
I_{3}(r)=\int_{r-R_{12}}^{r-\lambda} K I_{3} b(r, t) d t+\int_{r-\lambda}^{r+\lambda} K I_{3} a(r, t) d t \\
+\int_{r+\lambda}^{r+R_{12}} K I_{3} b(r, t) d t+\int_{\frac{D}{2}+\frac{R_{2}}{2}}^{\infty} K I_{3} c(r, t) d t
\end{array}
$$

where

$$
\begin{aligned}
& K I_{3} a(r, t)=2 \pi g_{2}(t) t^{2}\left(P_{12} J(\lambda,-1, r, t)+\right. \\
& \quad E_{1} J(\lambda, 0, r, t)+\left(P_{12}+F_{1}\right) J^{\prime}\left(\lambda, R_{12},-1, r, t\right)+ \\
& \quad F_{2} J^{\prime}\left(\lambda, R_{12}, 0, r, t\right)+F_{3} J^{\prime}\left(\lambda, R_{12}, 1, r, t\right)+ \\
& \left.\quad F_{4} J^{\prime}\left(\lambda, R_{12}, 3, r, t\right)\right)
\end{aligned}
$$

$$
\begin{gathered}
K I_{3} b(r, t)=2 \pi g_{2}(t) t^{2}\left(F_{2} J\left(R_{12}, 0, r, t\right)+\right. \\
F_{3} J\left(R_{12}, 1, r, t\right)+F_{4} J\left(R_{12}, 3, r, t\right)+ \\
\left.\left(P_{12}+F_{1}\right) J\left(R_{12},-1, r, t\right)\right)
\end{gathered}
$$

and

$$
K I_{3} c(r, t)=-2 \pi P_{12} g_{2}(t) \frac{t}{r}(r+t-|r-t|) .
$$

If

$$
\frac{D}{2}+\frac{R_{1}}{2} \leq r<\infty
$$

then

$$
\begin{aligned}
& I_{4}(r)=4 \pi\left(P_{12} \frac{\lambda^{2}}{2}+E_{1} \frac{\lambda^{3}}{3}+\right. \\
& \left(F_{1}+P_{12}\right) \frac{\left(R_{12}^{2}-\lambda^{2}\right)}{2}+F_{2} \frac{\left(R_{12}^{3}-\lambda^{3}\right)}{3}+ \\
& \left.F_{3} \frac{\left(R_{12}^{4}-\lambda^{4}\right)}{4}+F_{4} \frac{\left(R_{12}^{6}-\lambda^{6}\right)}{6}\right) .
\end{aligned}
$$

If

$$
\frac{D}{2}+\frac{R_{2}}{2} \leq r \leq \frac{D}{2}+\frac{R_{1}}{2}+R_{12},
$$

then

$$
\begin{gathered}
H_{1}(r)=\int_{\frac{D}{2}+\frac{R_{1}}{2}}^{r-\lambda} K H_{1} b(r, t) d t+\int_{r-\lambda}^{r+\lambda} K H_{1} a(r, t) d t \\
+\int_{r+\lambda}^{r+R_{12}} K H_{1} b(r, t) d t+\int_{\frac{D}{2}+\frac{R_{1}}{2}}^{\infty} K H_{1} c(r, t) d t
\end{gathered}
$$

and, if

$$
\frac{D}{2}+\frac{R_{1}}{2}+R_{12}<r<\infty
$$

then

$$
\begin{array}{r}
H_{1}(r)=\int_{r-R_{12}}^{r-\lambda} K H_{1} b(r, t) d t+\int_{r-\lambda}^{r+\lambda} K H_{1} a(r, t) d t \\
+\int_{r+\lambda}^{r+R_{12}} K H_{1} b(r, t) d t+\int_{\frac{D}{2}+\frac{R_{1}}{2}}^{\infty} K H_{1} c(r, t) d t
\end{array}
$$

where

$$
\begin{aligned}
& K H_{1} a(r, t)=2 \pi g_{1}(t) t^{2}\left(P_{12} J(\lambda,-1, r, t)+\right. \\
& \quad E_{1} J(\lambda, 0, r, t)+\left(P_{12}+F_{1}\right) J^{\prime}\left(\lambda, R_{12},-1, r, t\right)+ \\
& F_{2} J^{\prime}\left(\lambda, R_{12}, 0, r, t\right)+F_{3} J^{\prime}\left(\lambda, R_{12}, 1, r, t\right)+ \\
& \left.\quad F_{4} J^{\prime}\left(\lambda, R_{12}, 3, r, t\right)\right)
\end{aligned}
$$

$$
\begin{gathered}
K H_{1} b(r, t)=2 \pi g_{1}(t) t^{2}\left(F_{2} J\left(R_{12}, 0, r, t\right)+\right. \\
F_{3} J\left(R_{12}, 1, r, t\right)+F_{4} J\left(R_{12}, 3, r, t\right)+ \\
\left.\left(P_{12}+F_{1}\right) J\left(R_{12},-1, r, t\right)\right)
\end{gathered}
$$

and

$$
\begin{gathered}
K H_{1} c(r, t)=-2 \pi P_{12} g_{1}(t) \frac{t}{r}(r+t-|r-t|) . \\
\frac{D}{2}+\frac{R_{2}}{2} \leq r<\infty
\end{gathered}
$$$$
\text { If }
$$

then

$$
H_{2}(r)=I_{4}(r) .
$$


If

$$
\frac{D}{2}+\frac{R_{2}}{2} \leq r \leq \frac{D}{2}+\frac{3}{2} R_{2},
$$

then

$$
H_{3}(r)=\int_{\frac{D}{2}+\frac{R_{2}}{2}}^{r+R_{2}} K H_{3} a(r, t) d t+\int_{\frac{D}{2}+\frac{R_{2}}{2}}^{\infty} K H_{3} b(r, t) d t
$$

and, if

$$
\frac{D}{2}+\frac{3}{2} R_{2}<r<\infty
$$

then

$$
H_{3}(r)=\int_{r-R_{2}}^{r+R_{2}} K H_{3} a(r, t) d t+\int_{\frac{D}{2}+\frac{R_{2}}{2}}^{\infty} K H_{3} b(r, t) d t,
$$

where

$$
K H_{3} a(r, t)=2 \pi g_{2}(t) t^{2}\left(P_{22} J\left(R_{2},-1, r, t\right)+\right.
$$

$$
\begin{aligned}
& B_{1} J\left(R_{2}, 0, r, t\right)+B_{2} J\left(R_{2}, 1, r, t\right)+ \\
& \left.B_{3} J\left(R_{2}, 3, r, t\right)\right)
\end{aligned}
$$

and

$$
K H_{3} b(r, t)=-2 \pi P_{22} g_{2}(t) \frac{t}{r}(r+t-|r-t|) .
$$

Finally, if

$$
\frac{D}{2}+\frac{R_{2}}{2} \leq r<\infty
$$

then

$$
H_{4}(r)=4 \pi\left(\frac{P_{22} R_{2}^{2}}{2}+\frac{B_{1} R_{2}^{3}}{3}+\frac{B_{2} R_{2}^{4}}{4}+\frac{B_{3} R_{2}^{6}}{6}\right) .
$$




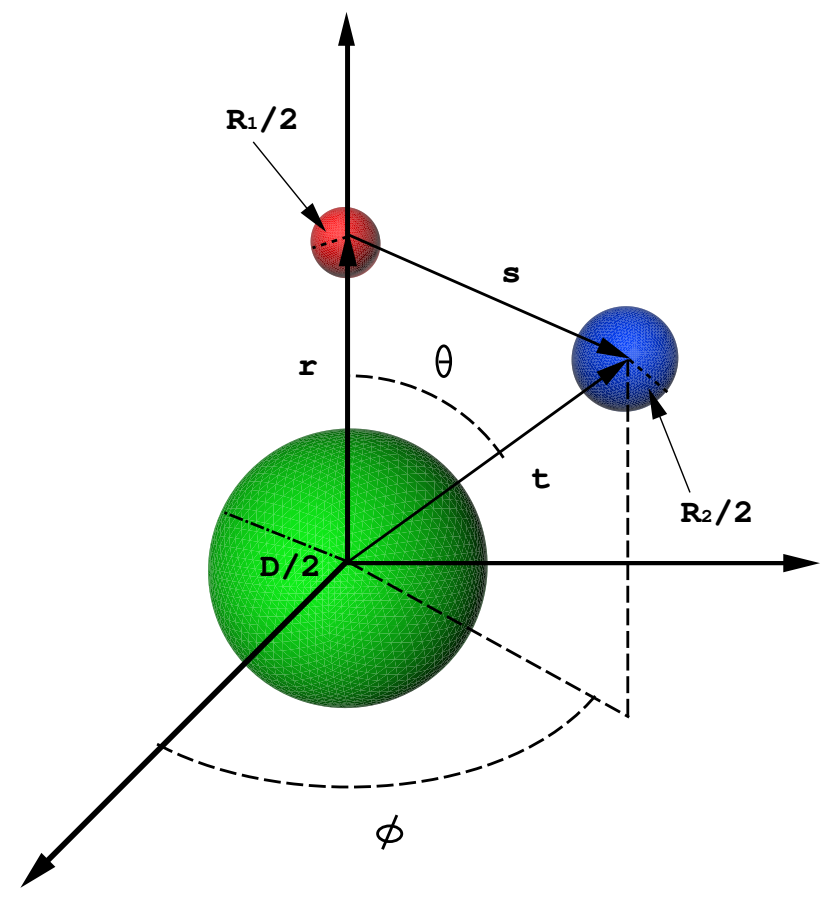

FIG. 1: Schematic representation of the model.

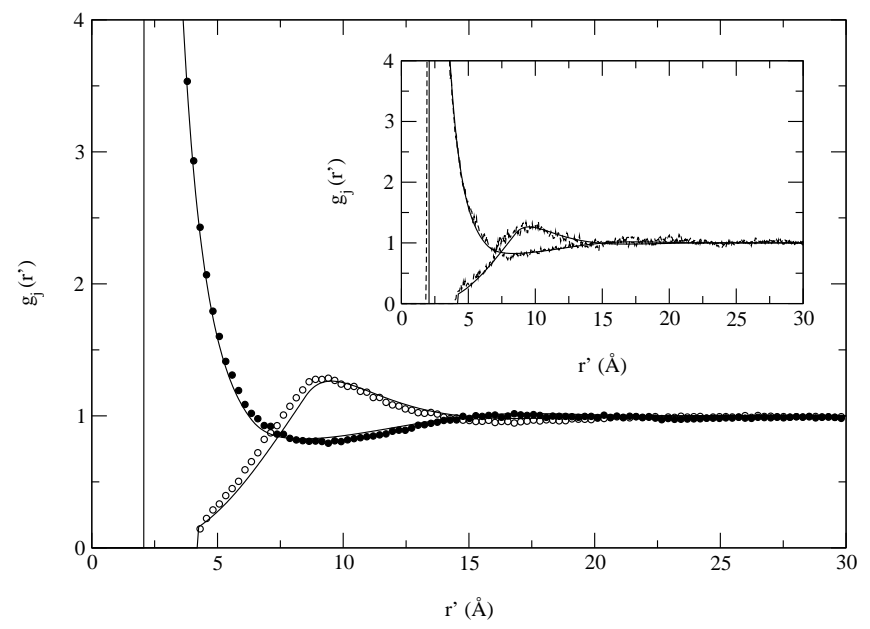

FIG. 2: SEDL radial distribution functions for a $2: 2,0.5 \mathrm{M}$ electrolyte around a colloid of diameter and surface charge density $D=10 \AA$ and $\sigma_{0}=0.407 \mathrm{C} / \mathrm{m}^{2}$, respectively. The ionic species have diameters $R_{-}=4.5 \AA$ and $R_{+}=8.5 \AA$. In the main panel the circles and the continuous lines correspond to Monte Carlo data and $\mathrm{HNC} / \mathrm{MSA}_{P M}$ results, respectively. In the inset the same system is considered, with the dashed and continuous lines corresponding to molecular dynamics simulations and to the $\mathrm{HNC} / \mathrm{MSA}_{P M}$ theory, respectively. The distance $r^{\prime}$ is measured from the macroparticle's surface. 


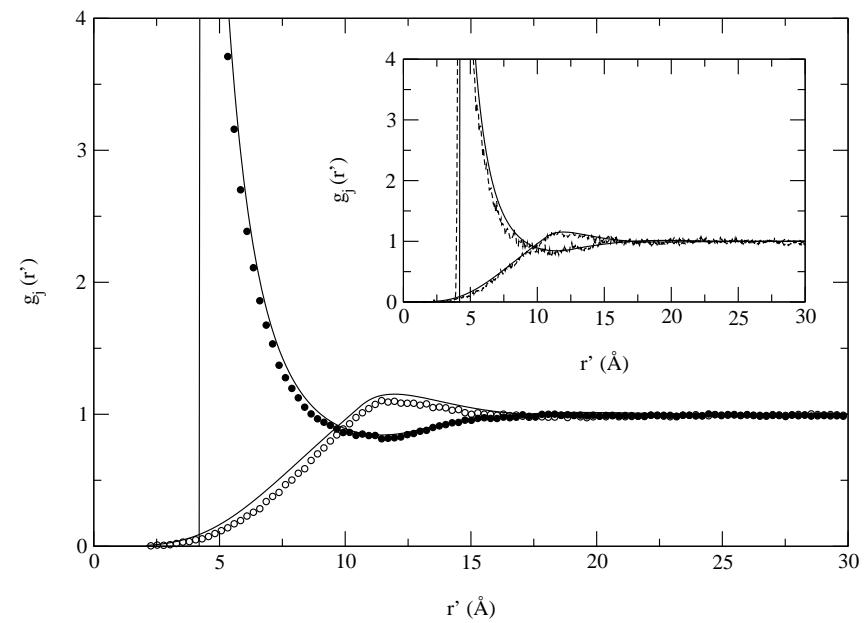

FIG. 3: SEDL radial distribution functions for a $2: 2,0.5 \mathrm{M}$ electrolyte around a colloid of diameter and surface charge density $D=10 \AA$ and $\sigma_{0}=0.407 \mathrm{C} / \mathrm{m}^{2}$, respectively. The ionic species have diameters $R_{-}=8.5 \AA$ and $R_{+}=4.25 \AA$. The symbols and curves have the same meaning as in Fig. 2

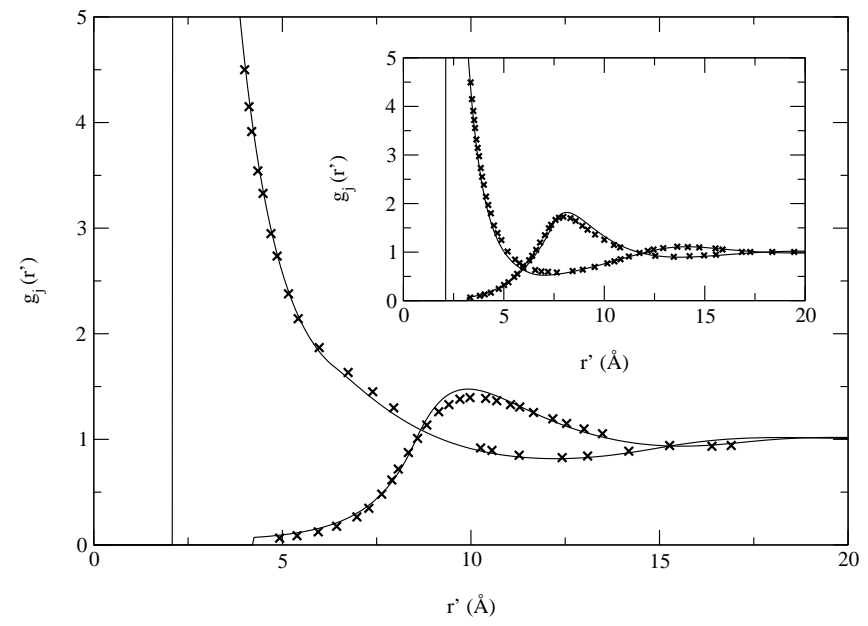

FIG. 4: HNC/MSA $P M$ and ARHNC radial distribution functions for monovalent and divalent $1 \mathrm{M}$ electrolytes. For $\mathrm{HNC} / \mathrm{MSA}_{P M}$ (continuous lines) the ionic distributions are around a very large colloid of diameter $D=1000 \times R_{-}$and surface charge density $\sigma_{0}=0.267 \mathrm{C} / \mathrm{m}^{2}$. For the ARHNC theory (with symbols) the profiles are next to a wall of the same charge $\sigma_{0}=0.267 \mathrm{C} / \mathrm{m}^{2}$. In the main panel the $1: 1$ ionic species have diameters $R_{-}=4.25 \AA$ and $R_{+}=8.5 \AA$ and in the inset the $2: 2$ ionic diameters are $R_{-}=4.25 \AA$ and $R_{+}=6.375 \AA$. The distance $r^{\prime}$ is measured from the charged surface. 

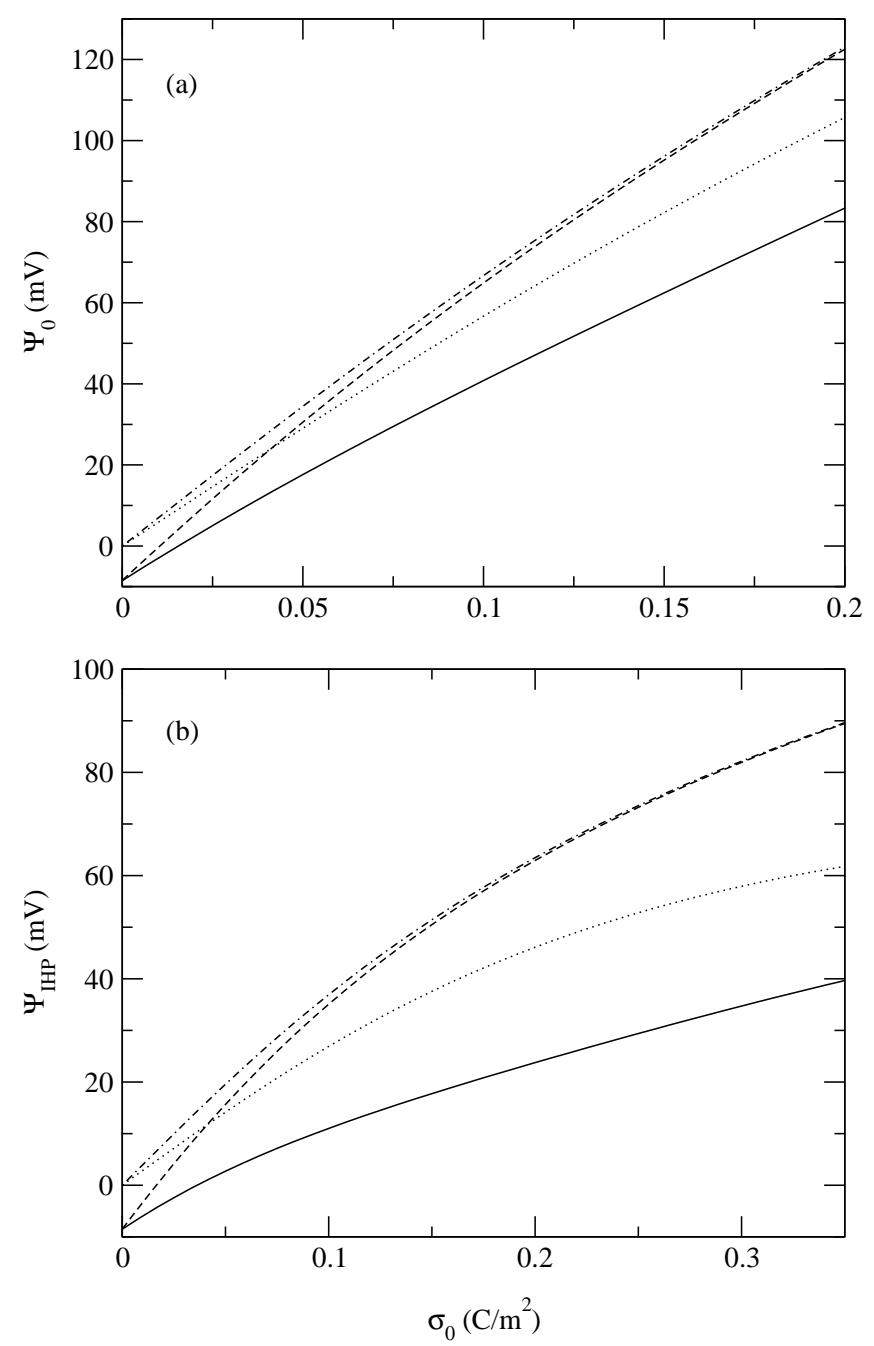

FIG. 5: SEDL mean electrostatic potential at: [a] the surface of the colloid and [b] the inner Helmholtz plane, as a function of the surface charge density $\sigma_{0}$, for a $1: 1,1 \mathrm{M}$ electrolyte with $R_{-}=4.25 \AA$ and $R_{+}=8.5 \AA$ around a macroion of diameter $D=160 \AA$ and non-negative surface charge density. The solid and dotted lines stand for HNC/MSA $P M$ and $\mathrm{HNC} / \mathrm{MSA}_{R P M}$, and the dashed and dot-dashed lines correspond to URMGC and MGC, respectively. The ioncolloid closest approach distances are: $d_{-}=\left(D+R_{-}\right) / 2$ and $d_{+}=\left(D+R_{+}\right) / 2$ for $\mathrm{HNC} / \mathrm{MSA}_{P M}$ and URMGC, and $d_{-}=$ $d_{+}=\left(D+R_{-}\right) / 2$ for HNC/MSA $R P M$ and MGC. The ion-ion closest approach distances are: $d_{-+}=d_{+-}=\left(R_{-}+R_{+}\right) / 2$ for $\mathrm{HNC} / \mathrm{MSA}_{P M}, d_{-+}=d_{+-}=R_{-}$for $\mathrm{HNC} / \mathrm{MSA}_{R P M}$, and $d_{-+}=d_{+-}=0$ for URMGC and MGC. 

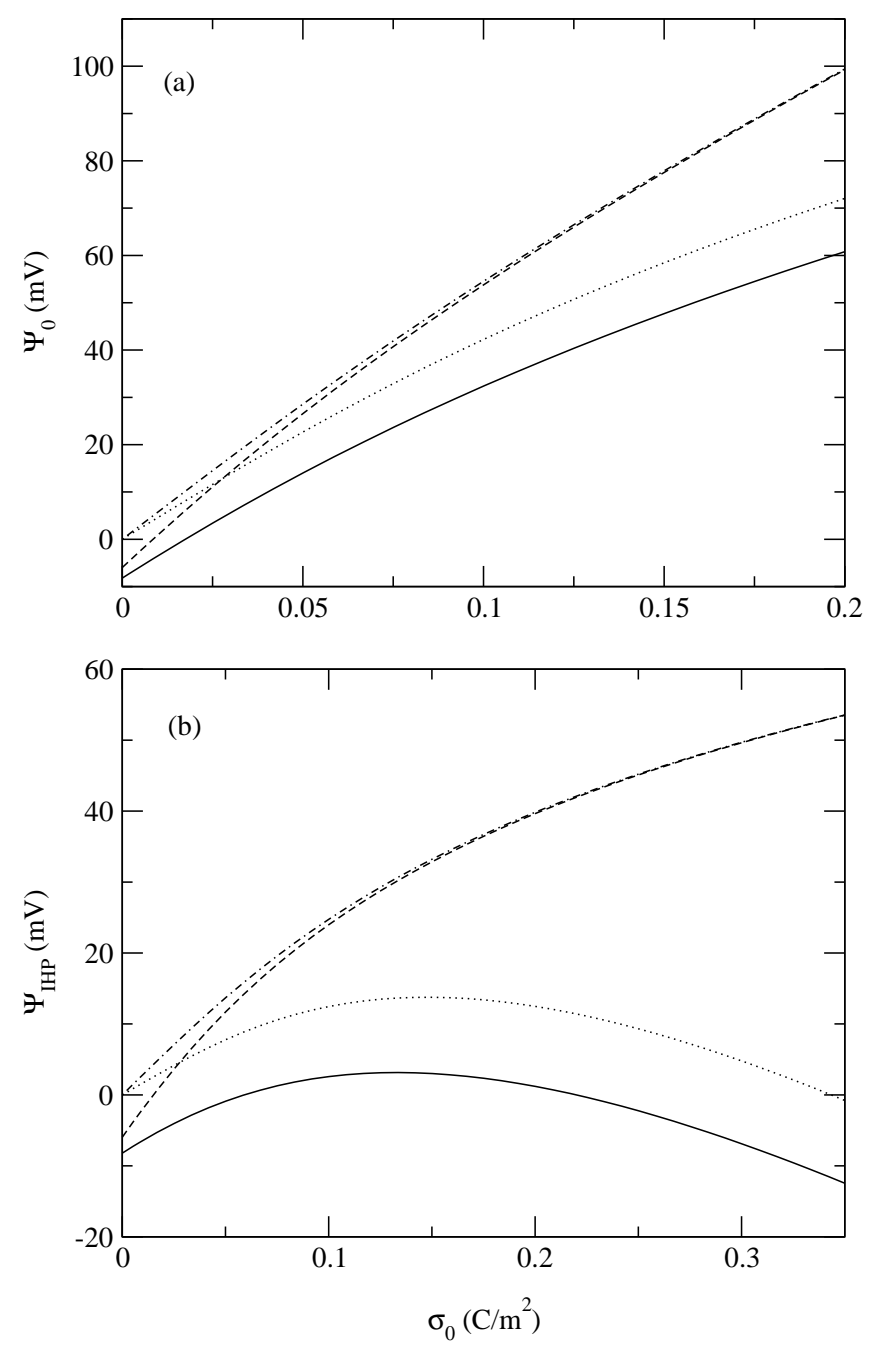

FIG. 6: The same as in Fig. [5 but for a 2:2, 0.5 M electrolyte.

TABLE I: Parameters of the 2:2, 0.5 electrolytes used in the Monte Carlo and molecular dynamics simulations of the asymmetric SEDL. A macroion with diameter $D=10 \AA$ and surface charge density $\sigma_{0}=0.407 \mathrm{C} / \mathrm{m}^{2}$ is employed in all the runs.

\begin{tabular}{cccccccccc}
\hline \hline Run & Method & $z_{1}$ & $z_{2}$ & $R_{1}(\AA)$ & $R_{2}(\AA)$ & $N_{1}$ & $N_{2}$ & $L$ \\
\hline $\mathrm{A}$ & $\mathrm{MC}$ & -2 & +2 & 4.25 & 8.50 & 1308 & 1304 & $38.35 R_{1}$ \\
$\mathrm{~B}$ & $\mathrm{DM}$ & -2 & +2 & 4.25 & 8.50 & 2894 & 2890 & 50.00 & $R_{1}$ \\
$\mathrm{C}$ & $\mathrm{MC}$ & +2 & -2 & 4.25 & 8.50 & 1304 & 1308 & 38.35 & $R_{1}$ \\
$\mathrm{D}$ & $\mathrm{DM}$ & +2 & -2 & 4.25 & 8.50 & 2890 & 2894 & 50.00 & $R_{1}$ \\
\hline \hline
\end{tabular}




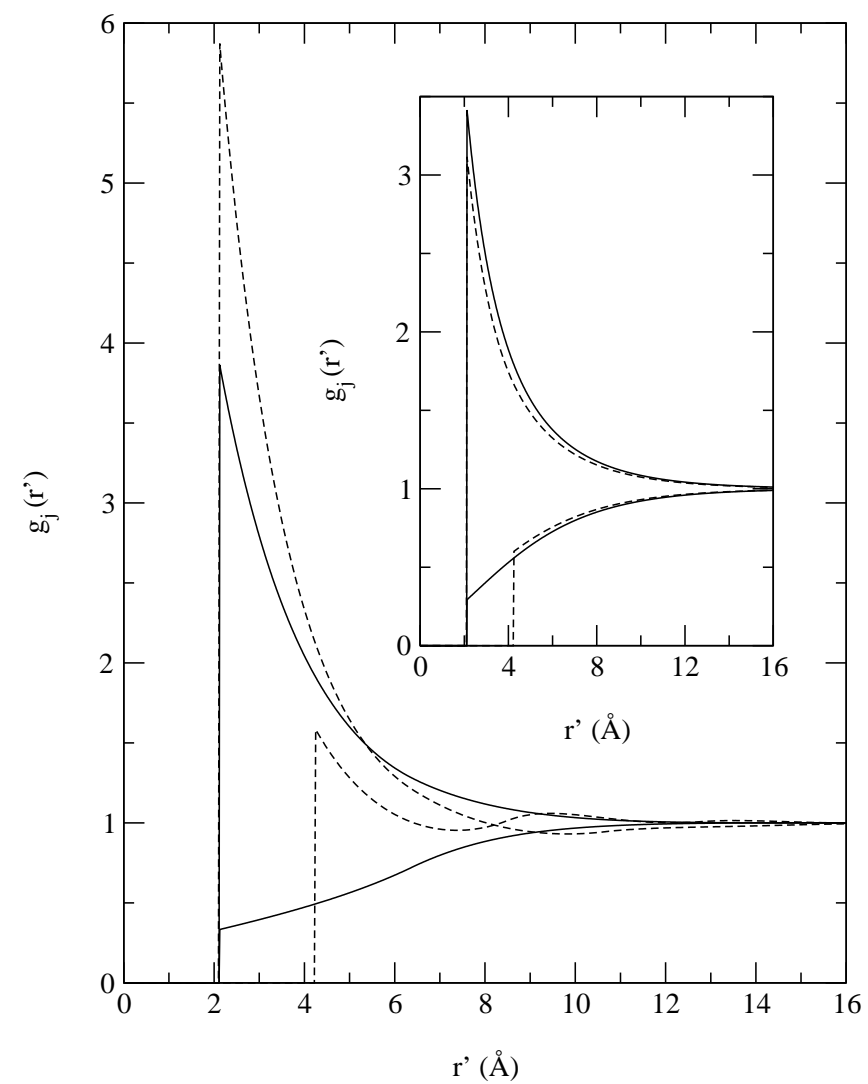

FIG. 7: SEDL radial distribution functions for a $1: 1,1 \mathrm{M}$ electrolyte with $R_{-}=4.25 \AA$ and $R_{+}=8.5 \AA$ next to a colloid of diameter $D=160 \AA$ and surface charge density $\sigma_{0}=$ $0.08356 \mathrm{C} / \mathrm{m}^{2}$. In the main panel the solid and dashed lines stand for $\mathrm{HNC} / \mathrm{MSA}_{R P M}$ and $\mathrm{HNC} / \mathrm{MSA}_{P M}$, respectively, whereas in the inset the solid and dashed lines correspond to MGC and URMGC, respectively. The colloid-ion and ion-ion closest approach distances used in each theory are the same as in Fig. [5 Here (and in the rest of figures) the distance $r^{\prime}$ is measured from the macroparticle's surface.

TABLE II: SEDL values of the radial distribution functions at $C_{-} \equiv\left(D+R_{-}\right) / 2$ and $C_{+} \equiv\left(D+R_{+}\right) / 2$ for $1: 1,1 \mathrm{M}$ and $2: 2$, $0.5 \mathrm{M}$ size-asymmetric electrolytes around a colloid of diameter $D=160 \AA$ and variable $\sigma_{0}$, obtained from the HNC/MSA $P M$, $\mathrm{HNC} / \mathrm{MSA}_{R P M}$, URMGC and MGC theories. The ionic species have diameters $R_{-}=4.25 \AA$ for the counterions and $R_{+}=8.5$ $\AA$ for the coions. The colloid-ion and ion-ion closest approach distances used in each theory are the same as in Fig. 5 The surface charge density $\sigma_{0}$ is in $C / m^{2}$.

\begin{tabular}{|c|c|c|c|c|c|c|c|c|c|}
\hline Electrolyte & $\sigma_{0}$ & $g_{-}^{\mathrm{HNC} / \mathrm{MSA}_{P M}}$ & $g_{+}^{\mathrm{HNC} / \mathrm{MSA}_{P M}}$ & $g_{-}^{\mathrm{HNC} / \mathrm{MSA}_{R P M}}$ & $g_{+}^{\mathrm{HNC}_{+} \mathrm{MSA}_{R P M}}$ & $g_{-}^{U R M G C}$ & $g_{+}^{U R M G C}$ & $g_{-}^{M G C}$ & $g_{+}^{M G C}$ \\
\hline $1: 1$ & 0.08356 & 5.87 & 1.6 & 3.7 & 0.3 & 3.1 & 0.6 & 3.4 & 0.3 \\
\hline $1: 1$ & 0.3004 & 28.8 & 0.06 & 25.4 & 0.01 & 24.4 & 0.3 & 24.6 & 0.04 \\
\hline $2: 2$ & 0.08356 & 6.2 & 0.5 & 5.6 & 0.2 & 4.9 & 0.6 & 5.3 & 0.2 \\
\hline $2: 2$ & 0.3004 & 50.7 & 0.03 & 49.7 & 0.009 & 47.8 & 0.3 & 48.1 & 0.02 \\
\hline
\end{tabular}




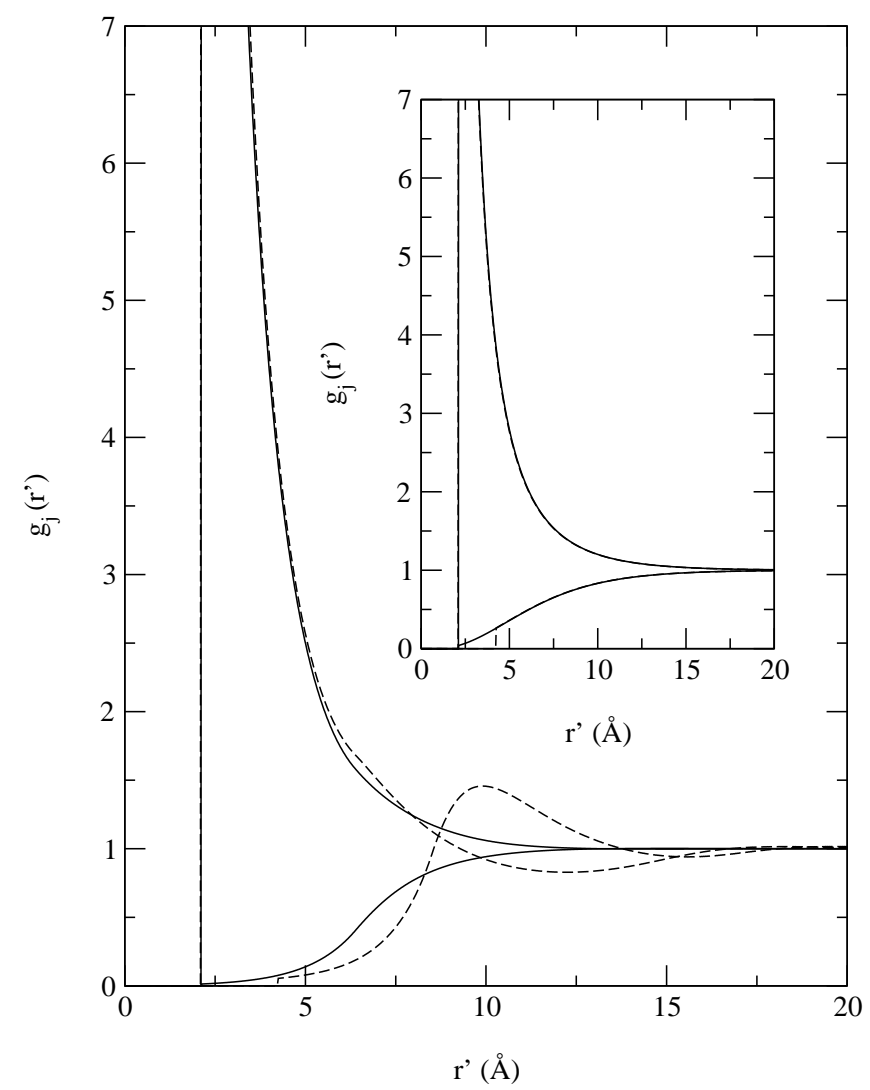

FIG. 8: The same as in Fig. [7 but for a surface charge density $\sigma_{0}=0.3004 \mathrm{C} / \mathrm{m}^{2}$.

TABLE III: SEDL mean electrostatic potentials at the macroion's surface $\left(\psi_{0}\right)$ and at the inner Helmholtz plane $\left(\psi_{I H P}\right)$ for the $\mathrm{HNC} / \mathrm{MSA}_{P M}, \mathrm{HNC} / \mathrm{MSA}_{R P M}$, URMGC and MGC theories. The parameters are the same as in Table II The surface charge density $\sigma_{0}$ is in $C / m^{2}$ and the MEPs in $m V$.

\begin{tabular}{|c|c|c|c|c|c|c|c|c|c|}
\hline Electrolyte & $\sigma_{0}$ & $\psi_{0}^{\mathrm{HNC} / \mathrm{MSA}_{P M}}$ & $\psi_{I H P}^{\mathrm{HNC} / \mathrm{MSA}_{P M}}$ & $\psi_{0}^{\mathrm{HNC} / \mathrm{MSA}_{R P M}}$ & $\psi_{I H P}^{\mathrm{HNC} / \mathrm{MSA}_{R P M}}$ & $\psi_{0}^{U R M G C}$ & $\psi_{I H P}^{U R M G C}$ & $\psi_{0}^{M G C}$ & $\psi_{I H P}^{M G C}$ \\
\hline $1: 1$ & 0.08356 & 33.4 & 8.5 & 47.8 & 22.9 & 54.1 & 29.2 & 56.4 & 31.5 \\
\hline $1: 1$ & 0.3004 & 124.3 & 34.8 & 147.4 & 58.0 & 171.5 & 82.0 & 171.7 & 82.2 \\
\hline $2: 2$ & 0.08356 & 26.7 & 1.8 & 36.2 & 11.3 & 45.3 & 20.4 & 46.3 & 21.4 \\
\hline $2: 2$ & 0.3004 & 82.6 & -6.9 & 94.2 & 4.8 & 139.2 & 49.7 & 139.2 & 49.7 \\
\hline
\end{tabular}




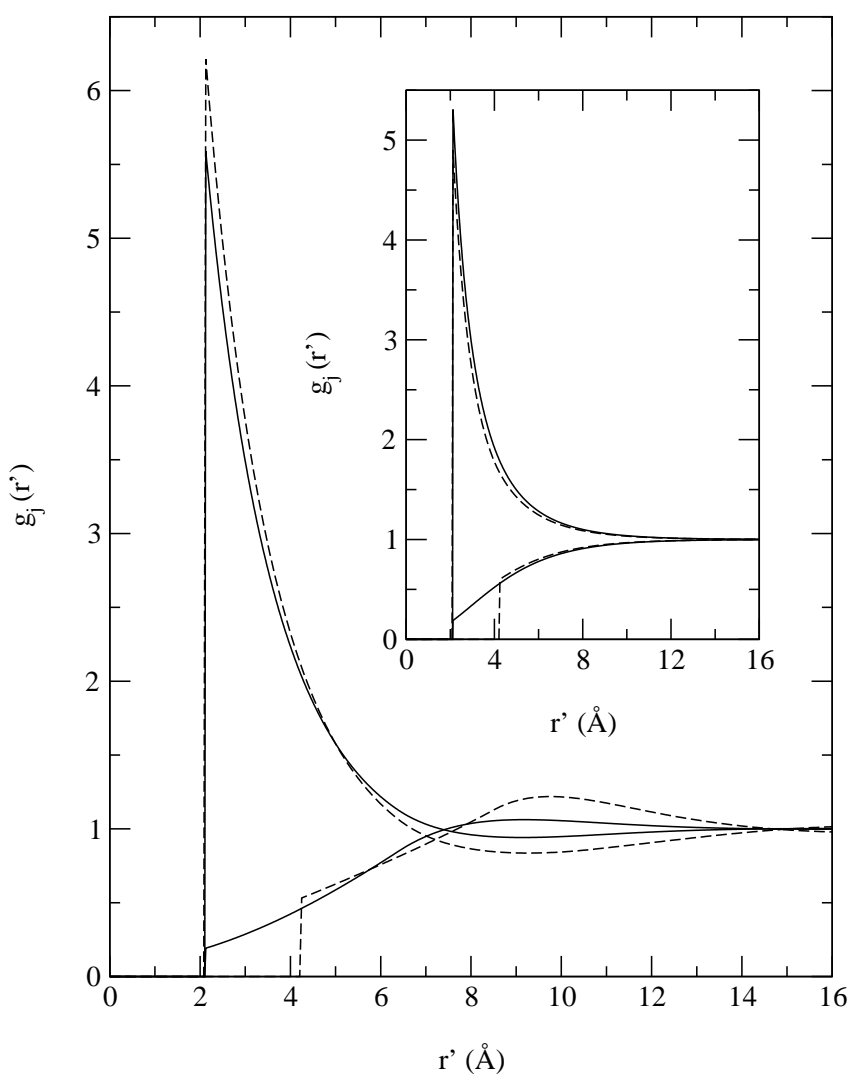

FIG. 9: SEDL radial distribution functions for a $2: 2,0.5 \mathrm{M}$ electrolyte with $R_{-}=4.25 \AA$ and $R_{+}=8.5 \AA$ next to a colloid of diameter $D=160 \AA$ and surface charge density $\sigma_{0}=$ $0.08356 \mathrm{C} / \mathrm{m}^{2}$. In the main panel the solid and dashed lines stand for $\mathrm{HNC} / \mathrm{MSA}_{R P M}$ and $\mathrm{HNC} / \mathrm{MSA}_{P M}$, respectively, whereas in the inset the solid and dashed lines correspond to MGC and URMGC, respectively. The colloid-ion and ion-ion closest approach distances used in each theory are the same as in Fig. [5] 


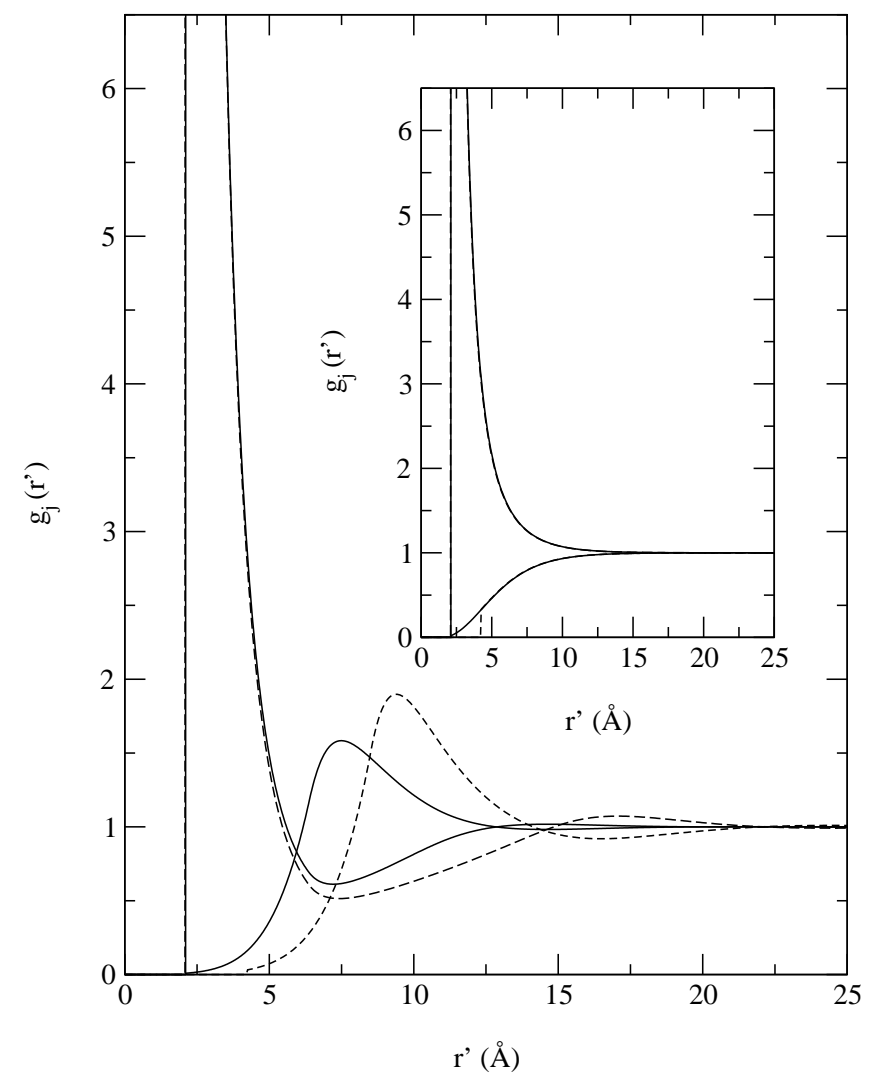

FIG. 10: The same as in Fig. 9 but for a surface charge density $\sigma_{0}=0.3004 \mathrm{C} / \mathrm{m}^{2}$. 


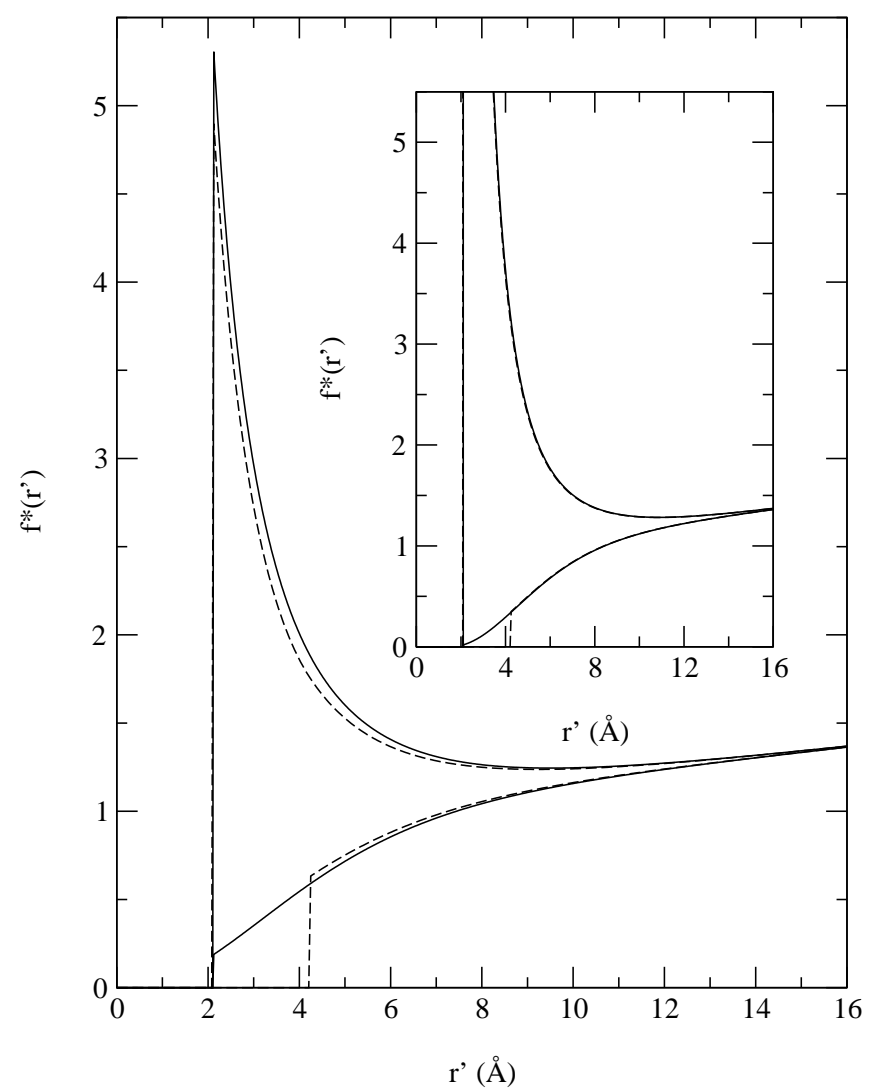

FIG. 11: SEDL reduced profiles $f^{*}\left(r^{\prime}\right)=f\left((D / 2)+r^{\prime}\right) / C_{-}^{2}$ for a $2: 2,0.5 \mathrm{M}$ with $R_{-}=4.25 \AA$ and $R_{+}=8.5 \AA$ around a colloid of diameter $D=160 \AA$. The solid and dashed lines are for MGC and URMGC, respectively. In the main panel the surface charge density is $\sigma_{0}=0.08356 \mathrm{C} / \mathrm{m}^{2}$, and in the inset is $\sigma_{0}=0.3004 \mathrm{C} / \mathrm{m}^{2}$. The colloid-ion and ion-ion closest approach distances used in each theory are the same as in Fig. 5 


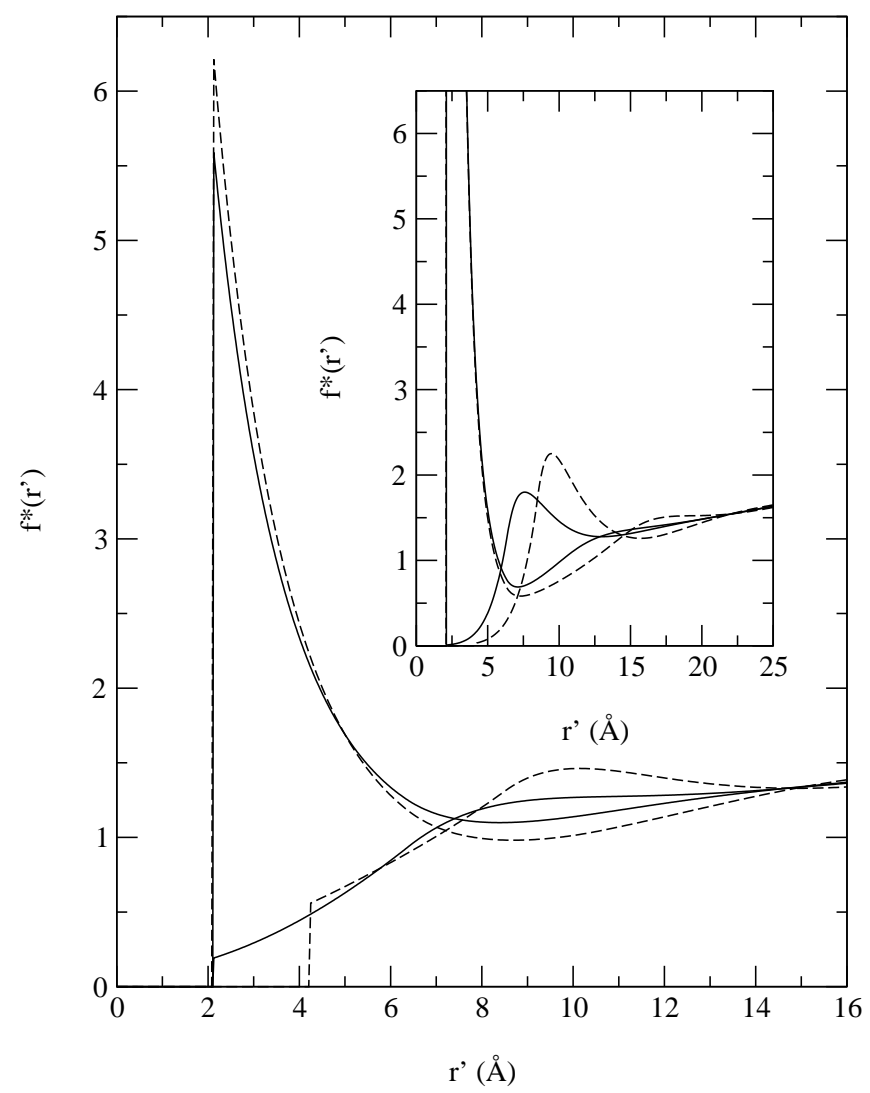

FIG. 12: SEDL reduced profiles $f^{*}\left(r^{\prime}\right)=f\left((D / 2)+r^{\prime}\right) / C_{-}^{2}$ for a 2:2, $0.5 \mathrm{M}$ with $R_{-}=4.25 \AA$ and $R_{+}=8.5 \AA$ around a colloid of diameter $D=160 \AA$. The solid and dashed lines are for $\mathrm{HNC} / \mathrm{MSA}_{R P M}$ and $\mathrm{HNC} / \mathrm{MSA}_{P M}$, respectively. In the main panel the surface charge density is $\sigma_{0}=0.08356 \mathrm{C} / \mathrm{m}^{2}$, and in the inset is $\sigma_{0}=0.3004 \mathrm{C} / \mathrm{m}^{2}$. The colloid-ion and ion-ion closest approach distances used in each theory are the

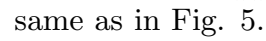




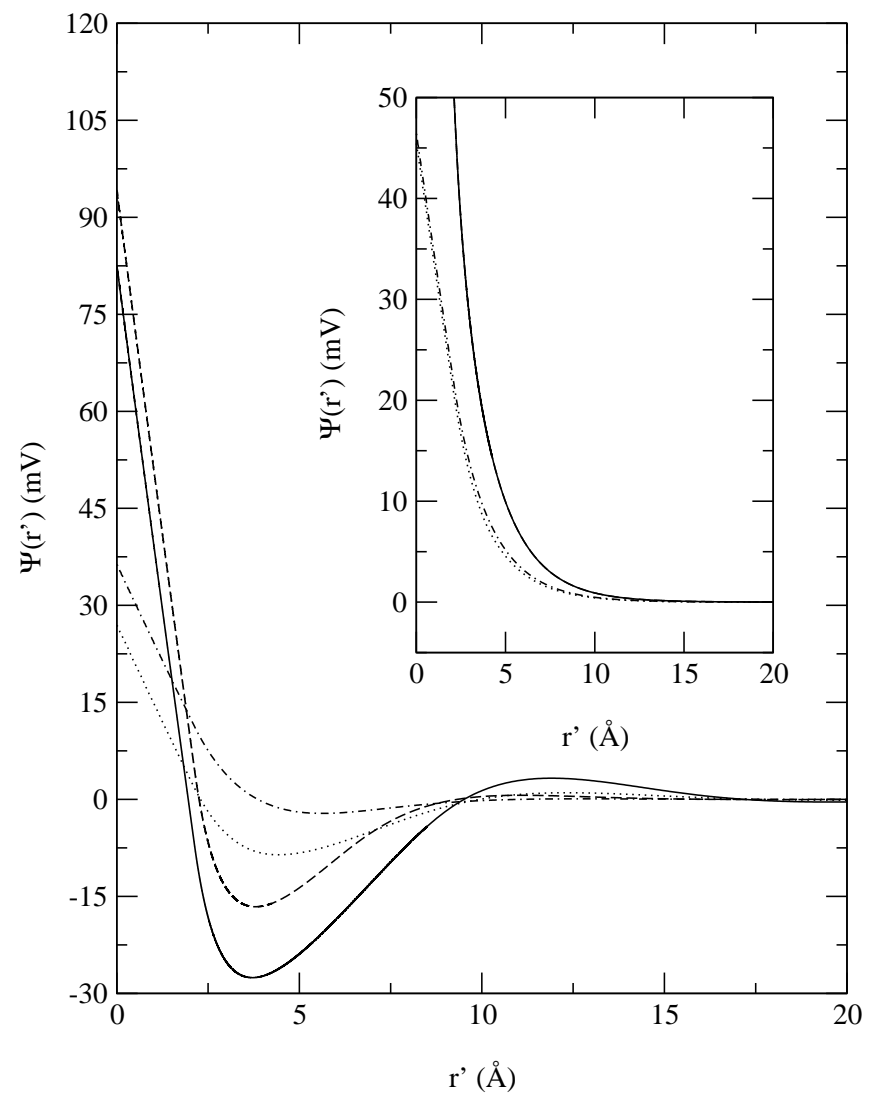

FIG. 13: SEDL mean electrostatic potential as a function of the distance to the macroparticle's surface for a $2: 2,0.5$ M with $R_{-}=4.25 \AA$ and $R_{+}=8.5 \AA$ around a colloid of diameter $D=160 \AA$. In the main panel the dotted line is for $\mathrm{HNC} / \mathrm{MSA}_{P M}$ and $\sigma_{0}=0.08356 \mathrm{C} / \mathrm{m}^{2}$, the solid line is for $\mathrm{HNC} / \mathrm{MSA}_{P M}$ and $\sigma_{0}=0.3004 \mathrm{C} / \mathrm{m}^{2}$, the dot-dashed is for $\mathrm{HNC} / \mathrm{MSA}_{R P M}$ and $\sigma_{0}=0.08356 \mathrm{C} / \mathrm{m}^{2}$, and the dashed line is for $\mathrm{HNC} / \mathrm{MSA}_{R P M}$ and $\sigma_{0}=0.3004 \mathrm{C} / \mathrm{m}^{2}$. In the inset the dotted line is for URMGC and $\sigma_{0}=0.08356 \mathrm{C} / \mathrm{m}^{2}$, the solid line is for URMGC and $\sigma_{0}=0.3004 \mathrm{C} / \mathrm{m}^{2}$, the dot-dashed is for MGC and $\sigma_{0}=0.08356 \mathrm{C} / \mathrm{m}^{2}$, and the dashed line is for MGC and $\sigma_{0}=0.3004 \mathrm{C} / \mathrm{m}^{2}$. The colloid-ion and ion-ion closest approach distances used in each theory are the same as in Fig. 5 


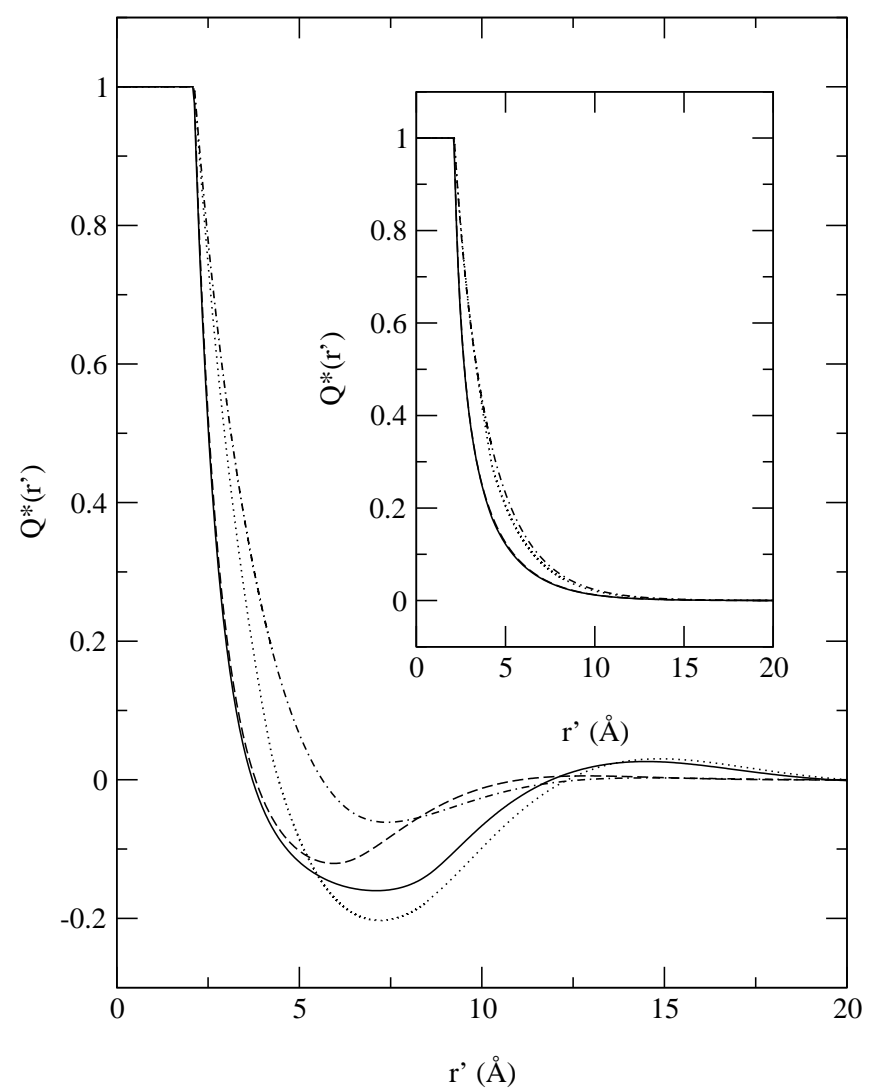

FIG. 14: SEDL cumulative reduced charge as a function of the distance to the macroparticle's surface for a $2: 2,0.5 \mathrm{M}$ with $R_{-}=4.25 \AA$ and $R_{+}=8.5 \AA$ around a colloid of diameter $D=160 \AA$. In the main panel the dotted line is for $\mathrm{HNC} / \mathrm{MSA}_{P M}$ and $\sigma_{0}=0.08356 \mathrm{C} / \mathrm{m}^{2}$, the solid line is for $\mathrm{HNC} / \mathrm{MSA}_{P M}$ and $\sigma_{0}=0.3004 \mathrm{C} / \mathrm{m}^{2}$, the dot-dashed is for $\mathrm{HNC} / \mathrm{MSA}_{R P M}$ and $\sigma_{0}=0.08356 \mathrm{C} / \mathrm{m}^{2}$, and the dashed line is for $\mathrm{HNC} / \mathrm{MSA}_{R P M}$ and $\sigma_{0}=0.3004 \mathrm{C} / \mathrm{m}^{2}$. In the inset the dotted line is for URMGC and $\sigma_{0}=0.08356 \mathrm{C} / \mathrm{m}^{2}$, the solid line is for URMGC and $\sigma_{0}=0.3004 \mathrm{C} / \mathrm{m}^{2}$, the dot-dashed is for MGC and $\sigma_{0}=0.08356 \mathrm{C} / \mathrm{m}^{2}$, and the dashed line is for MGC and $\sigma_{0}=0.3004 \mathrm{C} / \mathrm{m}^{2}$. The colloid-ion and ion-ion closest approach distances used in each theory are the same as in Fig. 5 